\title{
Evaluation of three lidar scanning strategies for turbulence measurements
}

\author{
Jennifer F. Newman ${ }^{1, \mathrm{a}}$, Petra M. Klein ${ }^{1}$, Sonia Wharton ${ }^{2}$, Ameya Sathe ${ }^{3, \mathrm{c}}$, Timothy A. Bonin ${ }^{1, \mathrm{~b}}$, Phillip B. Chilson ${ }^{1,4}$, \\ and Andreas Muschinski ${ }^{5}$ \\ ${ }^{1}$ School of Meteorology, University of Oklahoma, Norman, OK, USA \\ ${ }^{2}$ Atmospheric, Earth and Energy Division, Lawrence Livermore National Laboratory, Livermore, CA, USA \\ ${ }^{3}$ DTU Wind Energy, Risø Campus, Roskilde, Denmark \\ ${ }^{4}$ Advanced Radar Research Center, University of Oklahoma, Norman, OK, USA \\ ${ }^{5}$ NorthWest Research Associates, Boulder, CO, USA \\ anow at: National Wind Technology Center, National Renewable Energy Laboratory, Golden, CO, USA \\ ${ }^{b}$ now at: Cooperative Institute for Research in the Environmental Sciences, University of Colorado, and National Oceanic and \\ Atmospheric Administration/Earth System Research Laboratory, Boulder, CO, USA \\ c now at: DONG Energy, Copenhagen, Denmark
}

Correspondence to: Jennifer F. Newman (jennifer.newman@ nrel.gov)

Received: 23 October 2015 - Published in Atmos. Meas. Tech. Discuss.: 24 November 2015

Revised: 18 April 2016 - Accepted: 20 April 2016 - Published: 3 May 2016

\begin{abstract}
Several errors occur when a traditional Doppler beam swinging (DBS) or velocity-azimuth display (VAD) strategy is used to measure turbulence with a lidar. To mitigate some of these errors, a scanning strategy was recently developed which employs six beam positions to independently estimate the $u, v$, and $w$ velocity variances and covariances. In order to assess the ability of these different scanning techniques to measure turbulence, a Halo scanning lidar, WindCube v2 pulsed lidar, and ZephIR continuous wave lidar were deployed at field sites in Oklahoma and Colorado with collocated sonic anemometers.

Results indicate that the six-beam strategy mitigates some of the errors caused by VAD and DBS scans, but the strategy is strongly affected by errors in the variance measured at the different beam positions. The ZephIR and WindCube lidars overestimated horizontal variance values by over $60 \%$ under unstable conditions as a result of variance contamination, where additional variance components contaminate the true value of the variance. A correction method was developed for the WindCube lidar that uses variance calculated from the vertical beam position to reduce variance contamination in the $u$ and $v$ variance components. The correction method reduced WindCube variance estimates by over $20 \%$ at both the Oklahoma and Colorado sites under unstable con-
\end{abstract}

ditions, when variance contamination is largest. This correction method can be easily applied to other lidars that contain a vertical beam position and is a promising method for accurately estimating turbulence with commercially available lidars.

\section{Introduction}

Atmospheric turbulence, a measure of small-scale fluctuations in wind speed, impacts a number of fields, including air quality (e.g., Collier et al., 2005), aviation (e.g., Clark et al., 2000), and numerical weather prediction (e.g., Bright and Mullen, 2002). In particular, lidar-measured turbulence is a significant parameter in the wind energy industry, where high-resolution measurements are often needed in remote locations. Wind power production can differ substantially as a result of turbulence (e.g., Wharton and Lundquist, 2012; Clifton and Wagner, 2014), and turbulence can induce damaging loads on the turbine blades, reducing the turbine's reliability and expected lifetime (e.g., Kelley et al., 2006). Thus, turbulence is an extremely important parameter to measure in the wind farm site selection and design process. 
In the wind power industry, turbulence is typically estimated from cup anemometer measurements on meteorological towers. Measurements from cup anemometers are limited by tower height and can be plagued by issues with overspeeding and slow response times, which can lead to inaccurate mean wind speed and turbulence measurements (Kaimal and Finnigan, 1994). Sonic anemometers can measure turbulence much more accurately than cup anemometers but are also limited by tower height. In response to these issues, remote sensing devices, such as sodars (sound detection and ranging) and lidars (light detection and ranging), have recently emerged as a promising alternative to anemometers on towers. Although the ability of wind lidars to accurately measure mean horizontal wind speeds has been well-documented in the literature (e.g., Sjöholm et al., 2008; Pichugina et al., 2008; Peña et al., 2009; Barthelmie et al., 2013; Machta and Boquet, 2013; Sathe et al., 2015), the measurement of turbulence with lidars is still an active area of research (Sathe and Mann, 2013).

While cup anemometers measure wind speed at a small point in space, remote sensing devices report an average wind speed from a probe volume (typically $30-150 \mathrm{~m}$ in the vertical) and usually take measurements less frequently than tower-mounted instruments. These differences in spatial and temporal resolution lead to differences in the turbulence measured by cup anemometers and remote sensing devices (e.g., Peña et al., 2009; Westerhellweg et al., 2010; Krishnamurthy et al., 2014). While turbulent scales of motion can range from milliseconds to hours and from centimeters to kilometers (e.g., Stull, 2000), lidars are only capable of measuring turbulent motions with timescales on the order of seconds and spatial scales on the order of tens of meters. In addition to differences in spatial and temporal sampling, the scanning strategy used by the remote sensing device can also induce errors in the different turbulence components (Sathe and Mann, 2012).

Most commercially available lidars employ a Doppler beam swinging (DBS; Strauch et al., 1984) technique or a velocity-azimuth display (VAD; Browning and Wexler, 1968) technique to collect wind speed measurements. Using lidar DBS and VAD scans, the variances of the $u, v$, and $w$ velocity components are not directly measured; rather, the DBS and VAD techniques combine radial velocity measurements from different points around the scanning circle to calculate instantaneous values of the velocity components. The time series of $u, v$, and $w$ are then used to calculate the velocity variances, whereby it is implicitly assumed that the instantaneous velocity values are constant across the scanning circle. In turbulent flow, this assumption is not valid even if the mean flow is homogenous across the scanning circle, and the standard DBS and VAD approach for computing variances is thus flawed by variance contamination errors (Sathe and Mann, 2012). A different variance calculation approach was proposed by Sathe (2012) using a novel six-beam scanning technique, which utilizes the radial velocity variance values from six lidar beam positions to independently calculate the six unique components of the Reynolds stress tensor, i.e., the velocity variances and covariances.

Sathe et al. (2015) evaluated the six-beam technique at the Danish National Test Center for Large Wind Turbines in Høvsøre, Denmark, using the WindScanner lidar developed at Denmark Technical University. Sathe et al. (2015) found that the six-beam technique measured higher values of variance than the VAD technique for all stability classes, with values that were greater in magnitude and closest to the cup anemometer values under stable conditions. These findings are in contrast to observations presented by Sathe et al. (2011) for the same site, which indicates that lidars measure much larger values of variance under unstable conditions due to the larger turbulent motions present under these conditions. Sathe et al. (2015) attribute this difference to the wind directions selected for each of the studies; while only westerly wind directions were analyzed in the six-beam study, Sathe et al. (2011) analyzed only data that were associated with easterly wind directions. Since the WindScanner used by Sathe et al. (2015) was located $2 \mathrm{~km}$ east of the coast of the North Sea, data from the westerly wind direction could be influenced by the land-sea transition. As discussed by Sathe et al. (2015), this transition likely caused an internal boundary layer to develop, which, in conjunction with the current atmospheric stability regime, would affect the turbulent scales of motion intercepted by the lidar and the cup anemometer.

The six-beam technique, like the DBS and VAD techniques, is affected by volume averaging within the lidar probe volume. All three of these techniques also assume the three-dimensional flow is horizontally homogeneous across the scanning circle used by the lidar, which is often not a valid assumption (e.g., Wainwright et al., 2014; Lundquist et al., 2015), especially in complex terrain (e.g., Bingöl et al., 2009). All lidar scanning strategies are subject to sources of error, and the magnitude of these errors is largely dependent on atmospheric stability, measurement height, and the particular type of lidar used (e.g., Sathe et al., 2011). Wind energy developers and researchers must know how accurately their lidar can measure turbulence under different conditions if they want to use turbulence information for resource assessment or site suitability studies.

The main goals of this study are to evaluate the accuracy of lidar turbulence measurements and to provide guidance about lidar scanning strategies for wind energy applications. To this end, three main research questions are addressed in this work: (1) how well do two commonly used scanning strategies (the DBS and VAD techniques) measure turbulence under different stability conditions? (2) How well does the new six-beam technique measure turbulence under different stability conditions? (3) Can new data processing techniques reduce the errors in velocity variance calculations from lidar DBS scans? To address these questions, turbulence measured with the various techniques is compared to turbulence measured by three-dimensional sonic anemome- 
ters on tall towers at sites in Oklahoma and Colorado. Sonic anemometer data from the Boulder Atmospheric Observatory (BAO) in Colorado are used to quantify the additional terms that occur as a result of variance contamination and to develop improved data processing techniques that reduce variance contamination errors. Data from the Southern Great Plains Atmospheric Radiation Measurement (ARM) site in Oklahoma provide a second location to test the new processing techniques with the DBS scan.

To the authors' knowledge, this work represents the first time the six-beam technique has been experimentally validated with high-frequency sonic anemometers and commercially available lidars. The use of commercially available lidars allows for an evaluation of turbulence measured with lidar technologies and scanning strategies that are commonly employed in the wind energy industry.

\section{Lidar scanning strategies and estimation of turbulence parameters}

\subsection{Current lidar technology}

One frequently used lidar in the wind energy industry is the Leosphere WindCube lidar, a pulsed Doppler lidar that emits short pulses of laser energy to measure radial wind speed. The time series of the returned signal is then split up into blocks that correspond to range gates and processed to estimate the average radial wind speed at each range gate. The sign and magnitude of the radial wind speed are determined from the Doppler shift of the returned signal with respect to the original signal (Huffaker and Hardesty, 1996). The Leosphere WindCube v2 model was used in this work.

Another type of Doppler lidar using pulsed $1.5 \mu \mathrm{m}$ lasers is the Halo Streamline manufactured by Halo Photonics (Pearson et al., 2009). The Halo Streamline (thereafter referred to as Halo lidar) is a scanning lidar, which allows the user to configure and choose different types of scanning routines. In our study, the Halo was used to evaluate both a six-beam and a VAD scanning technique, which are further detailed in the next section.

Unlike the WindCube and Halo lidars, the ZephIR is a continuous wave lidar and focuses the laser beam at different heights to obtain wind speed measurements. The ZephIR must collect velocity measurements individually at each measurement height, so it takes approximately $15 \mathrm{~s}$ to complete a full volumetric scan with 10 measurement heights. The probe length of the focused ZephIR beam increases with height and, thus, the size of the range gates is not constant. (The probe length is approximately $10 \mathrm{~m}$ at a range of $100 \mathrm{~m}$, but much smaller closer to the ground; Slinger and Harris, 2012.) The ZephIR continuously receives backscattered radiation, so it can collect data at ranges as low as $10 \mathrm{~m}$. However, the ZephIR cannot determine the direction of the Doppler shift in the received time series, and there is a $180^{\circ}$ ambiguity in the wind direction. The ZephIR 300, which was used in this work, has an attached met station with wind direction measurements, which can provide an estimate for the remotely measured wind direction (Slinger and Harris, 2012). However, this estimated wind direction was not always accurate during our field campaign in Colorado, and wind direction information from the sonic anemometers had to be used to correct the ZephIR wind direction measurements.

\subsection{General conventions}

In this work, we follow standard meteorological conventions for $u, v$, and $w$, where $u$ is the east-west component ( $u>0$ for wind coming from the west), $v$ is the north-south component ( $v>0$ for wind coming from the south), and $w$ is the vertical component ( $w>0$ for upward motion). Lidar data are presented using a spherical coordinate system, where $\theta$ is the azimuthal angle of the lidar beam measured clockwise from true north and $\phi$ is the elevation angle of the lidar beam measured from the ground. The radial velocity, $v_{\mathrm{r}}$, measured by the lidar is defined as positive for motion away from the lidar and negative for motion toward the lidar.

All three lidar systems evaluated in this study use some variant of a plan-position indicator (PPI) scan to measure the three-dimensional wind components, where the lidar takes measurements at several azimuth angles around a scanning circle at a constant elevation angle. In a horizontally homogeneous atmosphere, the radial velocity values measured by a lidar completing a PPI scan should take the following form (Weitkamp, 2005):

$v_{\mathrm{r}}=u \sin \theta \cos \phi+v \cos \theta \cos \phi+w \sin \phi$.

When calculating velocity variances from Eq. (1), two different approaches can be used. The standard method is to apply DBS or VAD analysis techniques to the PPI data to compute instantaneous values of $u, v$, and $w$ for each time stamp. The variances are then computed using

$\overline{u_{i}^{\prime 2}}=\overline{\left(u_{i}(t)-\overline{u_{i}}\right)^{2}}$

where the index $i=1,2,3$ refers to the three velocity components $u, v$, and $w$ and the overbar denotes temporal averaging.

The second method involves first computing the variance of the radial velocities given by Eq. (1):

$$
\begin{aligned}
\overline{v_{\mathrm{r}}^{\prime 2}}= & \overline{u^{\prime 2}} \cos ^{2} \phi \sin ^{2} \theta+\overline{v^{\prime 2}} \cos ^{2} \phi \cos ^{2} \theta+\overline{w^{\prime 2}} \sin ^{2} \phi \\
& +2 \overline{u^{\prime} v^{\prime}} \cos ^{2} \phi \cos \theta \sin \theta+2 \overline{u^{\prime} w^{\prime}} \cos \phi \sin \phi \sin \theta \\
& +2 \overline{v^{\prime} w^{\prime}} \cos \phi \sin \phi \cos \theta .
\end{aligned}
$$

The variances and covariances of the velocity components $u, v$, and $w$ create a set of six unknown variables. By using six different beam positions (i.e., different combinations of $\theta$ and $\phi$ ), a set of equations can be solved for the six unknown variables (Sathe, 2012). 
The different lidar scanning and data analysis approaches for computing mean values and variances of $u, v$, and $w$ are discussed in more detail in the following sections. For reference, a schematic of the DBS and VAD scanning strategies can be found in Sect. 12.4.3 of Weitkamp (2005).

\subsection{WindCube v2: DBS technique}

The WindCube v2 measures wind speed with a DBS technique, where an optical switch is used to point the lidar beam in the four cardinal directions (north, east, south, and west) at an elevation angle of $62^{\circ}$ from the ground. Equations for the instantaneous radial velocities measured at the four beam positions can be derived by letting $\theta=0,90,180$, and $270^{\circ}$ in Eq. (1):

$v_{\mathrm{r} 1}=v_{1} \cos \phi+w_{1} \sin \phi$,

$v_{\mathrm{r} 2}=u_{2} \cos \phi+w_{2} \sin \phi$,

$v_{\mathrm{r} 3}=-v_{3} \cos \phi+w_{3} \sin \phi$,

$v_{\mathrm{r} 4}=-u_{4} \cos \phi+w_{4} \sin \phi$,

where $u_{j}, v_{j}$, and $w_{j}$ are the instantaneous values of the velocity components at the four beam positions, and the index $j=1,2,3$, and 4 describes the values measured by the north-, east-, south-, and west-pointing beams, respectively. Some WindCube lidars, including the model used here, add a vertically pointing beam position, $v_{\mathrm{r} 5}$, which provides a direct measurement of the vertical velocity, $w\left(v_{\mathrm{r} 5}=w_{5}\right)$. It takes the WindCube lidar $1 \mathrm{~s}$ to collect data at each beam location, and steer the beam to the next beam location such that a full DBS scan takes approximately 4-5 s. However, the WindCube velocity algorithm calculates the $u, v$, and $w$ components every second using the current radial velocity and the radial velocities obtained from the previous three beam locations (Peña et al., 2015).

In lidar studies, Eqs. (4)-(7) are usually solved for $u$, $v$, and $w$ assuming that the flow is homogenous, i.e., the mean values of the three-dimensional wind components do not change across the scanning circle (e.g., Peña et al., 2015). Letting $\overline{u_{2}}=\overline{u_{4}}=\bar{u}, \overline{v_{1}}=\overline{v_{3}}=\bar{v}$, and $\overline{w_{1}}=\overline{w_{2}}=$ $\overline{w_{3}}=\overline{w_{4}}=\bar{w}$, equations for the mean velocity values can be found:

$\bar{u}=\frac{\overline{v_{\mathrm{r} 2}}-\overline{v_{\mathrm{r} 4}}}{2 \cos \phi}$,

$\bar{v}=\frac{\overline{v_{\mathrm{r} 1}}-\overline{v_{\mathrm{r} 3}}}{2 \cos \phi}$,

$\bar{w}=\frac{P\left(\overline{v_{\mathrm{r} 1}}+\overline{v_{\mathrm{r} 3}}\right)+Q\left(\overline{v_{\mathrm{r} 2}}+\overline{v_{\mathrm{r} 4}}\right)}{2 \sin \phi}$,

where $P=\cos ^{2} \Theta, Q=\sin ^{2} \Theta$, and $\Theta$ (degrees) is the wind direction. The $\bar{w}$ equation is a slightly modified version of the true DBS solution and is used by Leosphere to calculate the $w$ velocity for the WindCube lidar (e.g., Sathe et al., 2011). If a fifth vertical beam is used, the mean value of the vertical velocity component can also be calculated as $\bar{w}=\overline{v_{\mathrm{r} 5}}$.
Equations (8)-(10) are derived assuming that the values of $u, v$, and $w$ remain constant across the scanning circle. While this assumption is valid when computing mean values in homogenous flow, instantaneous velocity values will be highly variable due to the nature of turbulent flow, and the computation of instantaneous velocity values with Eqs. (8)-(10) is inaccurate. However, the standard DBS velocity variance calculation method uses Eqs. (8)-(10) to compute instantaneous values of $u, v$, and $w$, which leads to the variance contamination errors discussed in the literature (Sathe and Mann, 2012).

\subsection{WindCube v2: novel method to reduce DBS variance contamination}

The errors associated with the standard DBS variance method can be illustrated by applying Reynolds decomposition to the instantaneous velocity values at each beam position. For the first and third beam positions, the following set of equations is obtained:

$v_{1}=\bar{v}+v_{1}^{\prime}$
$w_{1}=\bar{w}+w_{1}^{\prime}$
$v_{3}=\bar{v}+v_{3}^{\prime}=v_{1}-v_{1}^{\prime}+v_{3}^{\prime}$
$w_{3}=\bar{w}+w_{3}^{\prime}=w_{1}-w_{1}^{\prime}+w_{3}^{\prime}$,

where the mean values $\bar{v}$ and $\bar{w}$ can be assumed to be constant across the scanning circle but the turbulent velocity fluctuations will differ $\left(v_{1}^{\prime} \neq v_{3}^{\prime}\right.$ and $\left.w_{1}^{\prime} \neq w_{3}^{\prime}\right)$. Combining Eqs. (4)-(7), an equation for the instantaneous velocity at beam position 1 can then be derived:

$v_{1}=\frac{v_{\mathrm{r} 1}-v_{\mathrm{r} 3}-\mathrm{d} v \cos \phi+\mathrm{d} w \sin \phi}{2 \cos \phi}$,

where $\mathrm{d} v=v_{3}^{\prime}-v_{1}^{\prime}$ and $\mathrm{d} w=w_{3}^{\prime}-w_{1}^{\prime}$. Comparing Eqs. (15) and (9) illustrates how turbulent fluctuations at the different beam positions, reflected by nonzero values of $\mathrm{d} v$ and $\mathrm{d} w$, affect the computation of instantaneous velocity values.

Taking the variance of Eq. (15) gives the following equation:

$$
\begin{aligned}
\overline{v_{1}^{\prime 2}}= & \frac{1}{4 \cos ^{2} \phi} \overline{\left(v_{\mathrm{r} 1}^{\prime}\right.}+\overline{v_{\mathrm{r} 3}^{\prime 2}}-2 \overline{v_{\mathrm{r} 1}^{\prime} v_{\mathrm{r} 3}^{\prime}}-2 \overline{v_{\mathrm{r} 1}^{\prime} \mathrm{d} v} \cos \phi \\
& +2 \overline{v_{\mathrm{r} 1}^{\prime} \mathrm{d} w} \sin \phi+2 \overline{v_{\mathrm{r} 3}^{\prime} \mathrm{d} v} \cos \phi \\
& -2 \overline{v_{\mathrm{r} 3}^{\prime} \mathrm{d} w} \sin \phi+\overline{\mathrm{d} v^{2}} \cos ^{2} \phi-2 \overline{\mathrm{d} v \mathrm{~d} w} \sin \phi \cos \phi \\
& \left.+\overline{\mathrm{d} w^{2}} \sin ^{2} \phi\right) .
\end{aligned}
$$

The terms involving $\mathrm{d} v$ and $\mathrm{d} w$ appear because data are being combined from two different beam positions to estimate the $v$ variance. These terms can be further modified by taking into account that $\mathrm{d} v=v_{3}^{\prime}-v_{1}^{\prime}$ and $\mathrm{d} w=w_{3}^{\prime}-w_{1}^{\prime}$. For homogeneous flow, we can assume that time-averaged correlations between different velocity components (i.e., turbulent fluxes) 
become independent of the position along the scanning circle as long as both components are measured at the same location; thus, $\overline{v_{1}^{\prime} w_{1}^{\prime}}=\overline{v_{3}^{\prime} w_{3}^{\prime}}=\overline{v^{\prime} w^{\prime}}, \overline{v_{1}^{\prime} v_{1}^{\prime}}=\overline{v_{3}^{\prime} v_{3}^{\prime}}=\overline{v^{\prime 2}}$, etc. If the velocity components are measured at different beam positions, the fluxes can be expressed using autocorrelation functions:

$\overline{v_{3}^{\prime} w_{1}^{\prime}}=\overline{v_{1}^{\prime} w_{3}^{\prime}}=\rho_{v w} \overline{v^{\prime} w^{\prime}}$

$\overline{v_{3}^{\prime} v_{1}^{\prime}}=\overline{v_{1}^{\prime} v_{3}^{\prime}}=\rho_{v} \overline{v^{\prime 2}}$

$\overline{w_{3}^{\prime} w_{1}^{\prime}}=\overline{w_{1}^{\prime} w_{3}^{\prime}}=\rho_{w} \overline{w^{\prime 2}}$,

whereby the autocorrelation functions of the different velocity components describe the spatial and temporal correlation of the instantaneous velocity components measured at beam positions 1 and 3. Equation 16 then becomes the following:

$$
\begin{gathered}
\overline{v_{1}^{\prime 2}=} \overline{\overline{v^{\prime 2}}}=\frac{1}{4 \cos ^{2} \phi}\left[\overline{v_{\mathrm{r} 1}^{\prime 2}}+\overline{{v_{\mathrm{r} 3}^{\prime}}^{2}}-2 \overline{v_{\mathrm{r} 1}^{\prime} v_{\mathrm{r} 3}^{\prime}}+2 \cos ^{2}\right. \\
\left.\phi \overline{v^{\prime 2}}\left(1-\rho_{v}\right)-2 \sin ^{2} \phi \overline{w^{\prime 2}}\left(1-\rho_{w}\right)\right]
\end{gathered}
$$

Equation (20) can then be solved for $\overline{v^{\prime 2}}$ to give a general expression for the actual $v$ variance that is measured when data are combined from different beam positions in the DBS technique:

$$
\begin{aligned}
\overline{v^{\prime 2}}= & \frac{2}{\left(1+\rho_{v}\right)} \frac{1}{4 \cos ^{2} \phi}\left[\overline{v_{\mathrm{r} 1}^{\prime 2}}+\overline{v_{\mathrm{r} 3}^{\prime 2}}-2 \overline{v_{\mathrm{r} 1}^{\prime} v_{\mathrm{r} 3}^{\prime}}\right] \\
& -\frac{\left(1-\rho_{w}\right) \sin ^{2} \phi}{\left(1+\rho_{v}\right) \cos ^{2} \phi} \overline{w^{\prime 2}} .
\end{aligned}
$$

When we apply the standard DBS approach we inherently assume that the velocity fluctuations at the different beam positions are the same, i.e., we assume that $\rho_{v}=1$ and $\rho_{w}=1$. Thus, the DBS equation for the $v$ variance becomes

$\overline{v_{\mathrm{DBS}}^{\prime 2}}=\frac{1}{4 \cos ^{2} \phi}\left[\overline{v_{\mathrm{r} 1}^{\prime 2}}+\overline{{v_{\mathrm{r} 3}^{\prime}}^{2}}-2 \overline{v_{\mathrm{r} 1}^{\prime} v_{\mathrm{r} 3}^{\prime}}\right]$.

Equation (21) can thus be recast into

$\overline{v^{\prime 2}}=\frac{2}{1+\rho_{v}} \overline{v_{\mathrm{DBS}}^{\prime}}-\frac{\left(1-\rho_{w}\right) \sin ^{2} \phi}{\left(1+\rho_{v}\right) \cos ^{2} \phi} \overline{w^{\prime 2}}$.

Similarly, an equation for the actual $u$ variance given in terms of the DBS variance can be derived:

$\overline{u^{\prime 2}}=\frac{2}{1+\rho_{u}} \overline{u_{\mathrm{DBS}}^{\prime}}-\frac{\left(1-\rho_{w}\right) \sin ^{2} \phi}{\left(1+\rho_{u}\right) \cos ^{2} \phi} \overline{w^{\prime 2}}$.

Given the actual spatial separation and time shift between different lidar beams, the autocorrelation function values are all less than 1 and the correction terms in Eqs. (23)-(24) may become significant. In particular, the second term in Eqs. (23)-(24) contains the ratio $\sin ^{2} \phi / \cos ^{2} \phi$, which is approximately equal to 3.54 for the WindCube v2 elevation angle of $62^{\circ}$. This illustrates that ignoring the contribution of fluctuations in the instantaneous velocity for the WindCube v2 can lead to a large overestimation of the horizontal velocity variances during convective conditions when $\overline{w^{\prime 2}}$ is large. The actual values of the autocorrelation functions will depend on atmospheric stability and wind speed, which complicates applying corrections to the DBS variance calculations. However, Eqs. (23) and (24) provide the advantage that the variances of $u$ and $v$ computed from the DBS equations can be corrected if the vertical velocity component is measured with a direct vertical beam, as was the case in our study, and estimates of the autocorrelation functions can be made.

In Sect. 5.1, sonic anemometer and lidar measurements are used to evaluate the autocorrelation functions, and the feasibility of applying the correction algorithm (Eqs. 23-24) under a range of different stability conditions is discussed.

\subsection{ZephIR 300: VAD technique}

The ZephIR lidar employs a rotating mirror to conduct a 50point VAD scan at each measurement height, using a similar elevation angle to the WindCube lidar $\left(\phi=60^{\circ}\right.$ for the ZephIR compared to $\phi=62^{\circ}$ for the WindCube lidar). For the VAD technique, the radial velocities measured by the instrument should create a rectified cosine curve as a function of azimuth angle (Lhermitte and Atlas, 1961), as in Eq. (1). In a standard VAD analysis, the curve is assumed to fit the following equation:

$v_{\mathrm{r}}(\theta)=a+b \cos \left(\theta-\theta_{\max }\right)$,

where $\theta$ (degrees) is the azimuthal angle of the lidar beam, $a$ $\left(\mathrm{m} \mathrm{s}^{-1}\right)$ is the offset of the curve from the zero-velocity line, $b\left(\mathrm{~ms}^{-1}\right)$ is the amplitude of the curve, and $\theta_{\max }$ (degrees) is the phase shift of the curve. Assuming a homogeneous flow field with no convergence or divergence, the horizontal wind speed, wind direction, and vertical wind speed are then derived from the following relations:

$v_{\mathrm{h}}=\frac{b}{\cos (\phi)}$,
$W D=\theta_{\max }$,
$w=\frac{a}{\sin (\phi)}$,

where $a, b$, and $\theta_{\max }$ are typically determined from a leastsquares approach. The values of $u$ and $v$ can then be derived from the horizontal wind speed, $v_{\mathrm{h}}$, and the wind direction.

Equations (26)-(28) are derived from the first-order coefficients of a Fourier decomposition of the radial velocity field, while higher-order terms in the Fourier decomposition are related to divergence and deformation (Browning and Wexler, 
Table 1. Overview of instruments used to evaluate different scanning strategies during LABLE 2 and LATTE.

\begin{tabular}{|c|c|c|c|c|c|}
\hline \multicolumn{6}{|c|}{ Campaign instrumentation } \\
\hline WindCube v2 & LABLE 2 & $40-200 \mathrm{~m}$ & $1 \mathrm{~Hz}$ & DBS & LLNL \\
\hline $\begin{array}{l}\text { ZephIR } 300 \\
\text { Continuous wave Doppler lidar }\end{array}$ & LATTE & $\begin{array}{l}10-200 \mathrm{~m} \\
10 \text { measurement heights } \\
\text { Variable range gate size } \\
(0.1-44 \mathrm{~m})\end{array}$ & $\begin{array}{l}0.07 \mathrm{~Hz} \\
\text { Full scan: } 15 \mathrm{~s}\end{array}$ & $\begin{array}{l}\text { VAD } \\
60^{\circ} \text { elevation angle }\end{array}$ & LLNL \\
\hline $\begin{array}{l}\text { Halo Streamline Pro } \\
\text { Scanning Doppler lidar }\end{array}$ & $\begin{array}{l}\text { LABLE } 2 \\
\text { LATTE }\end{array}$ & $\begin{array}{l}105 \mathrm{~m}-9.6 \mathrm{~km} \\
30 \mathrm{~m} \text { range gates }\end{array}$ & $\begin{array}{l}1 \mathrm{~Hz} \\
\text { Full scan: } 30 \mathrm{~s}\end{array}$ & $\begin{array}{l}\text { Six-beam } \\
45^{\circ} \text { elevation angle }\end{array}$ & OU \\
\hline $\begin{array}{l}\text { Gill Windmaster Pro } \\
\text { Three-dimensional sonic anemometer }\end{array}$ & LABLE 2 & $60 \mathrm{~m}$ & $10 \mathrm{~Hz}$ & - & $\begin{array}{l}\text { Lawrence } \\
\text { Berkeley } \\
\text { National } \\
\text { Laboratory }\end{array}$ \\
\hline $\begin{array}{l}\text { Campbell Scientific CSAT3 } \\
\text { Three-dimensional sonic anemometers }\end{array}$ & LATTE & $\begin{array}{l}50,100,150,200,250, \\
\text { and } 300 \mathrm{~m}, \mathrm{SE} \text { booms }\end{array}$ & $60 \mathrm{~Hz}$ & - & NCAR \\
\hline
\end{tabular}

1968). Although these higher-order terms are typically ignored in VAD analysis of lidar data, neglecting the terms can lead to errors in the estimated wind speed and direction (e.g., Koscielny et al., 1984). Errors in the turbulent components can arise as a result of variance contamination. Similar to the DBS technique, the VAD technique involves combining data from different beam positions with the assumption that the instantaneous velocity field is homogeneous across the scanning circle.

\subsection{Six-beam technique}

As discussed in the previous two sections, the use of either the DBS or the VAD technique introduces a number of known systematic errors into lidar turbulence calculations. Some of these errors can be mitigated when applying the second variance calculation method (Eq. 3), which involves solving a set of equations for different combinations of $\theta$ and $\phi$ to obtain all six components of the covariance matrix.

In this work, the six-beam technique developed by Sathe (2012) was evaluated using the user-configurable Halo lidar. Sathe (2012) developed the technique by using a minimization algorithm to determine the optimum combination of $\theta$ and $\phi$ values that minimizes the random errors in the variance estimates. The optimal configuration found was as follows: five beams at an elevation angle of $45^{\circ}$ that are equally spaced $72^{\circ}$ apart (i.e., located at azimuths of $0,72,144,216$, and $288^{\circ}$ ) and one vertically pointed beam. This scanning strategy is hereafter referred to as the six-beam technique.

Solving Eq. (3) with the chosen values of $\theta$ and $\phi$, the equations for the variances $\overline{u^{\prime 2}}, \overline{v^{\prime 2}}$, and $\overline{w^{\prime 2}}$ based on the six-beam technique are

$$
\begin{aligned}
& \overline{u_{6 b}^{\prime}{ }^{2}}=-0.4 \overline{{v_{\mathrm{r} 1}^{\prime}}^{2}}+1.05\left(\overline{{v_{\mathrm{r} 2}^{\prime}}^{2}}+\overline{{v_{\mathrm{r} 5}^{\prime}}^{2}}\right) \\
& +0.15\left(\overline{v_{\mathrm{r} 3}^{\prime 2}}+\overline{v_{\mathrm{r} 4}^{\prime 2}}\right)-\overline{{v_{\mathrm{r} 6}^{\prime}}^{2}}, \\
& \overline{v_{6 b}^{\prime}{ }^{2}}=1.2 \overline{{v_{\mathrm{r} 1}^{\prime}}^{2}}-0.25\left(\overline{{v_{\mathrm{r} 2}^{\prime 2}}^{2}}+\overline{{v_{\mathrm{r} 5}^{\prime}}^{2}}\right) \\
& +0.65\left(\overline{v_{\mathrm{r} 3}^{\prime 2}}+\overline{v_{\mathrm{r} 4}^{\prime 2}}\right)-\overline{{v_{\mathrm{r} 6}^{\prime}}^{2}}, \\
& \overline{w_{6 b}^{\prime}}=\overline{v_{\mathrm{r} 6}^{\prime 2}} \text {, }
\end{aligned}
$$

where subscript $6 b$ indicates that the horizontal velocity variances are computed applying the six-beam technique, and subscripts 1-6 refer to the beam positions, with beams 1-5 spaced $72^{\circ}$ apart in the scanning circle and beam 6 pointing vertically upward.

\section{Measurement campaigns}

The DBS and six-beam strategies were evaluated at a field site in Oklahoma, while all three scanning strategies were evaluated at a field site in Colorado. As the Colorado site featured a large amount of three-dimensional sonic anemometer verification data, this site will be described first and will be primarily used to draw conclusions about the accuracy of lidar turbulence measurements. These results will be corroborated by data collected during the Oklahoma experiment. Instruments used to evaluate the various scanning techniques are summarized in Table 1. 
a)

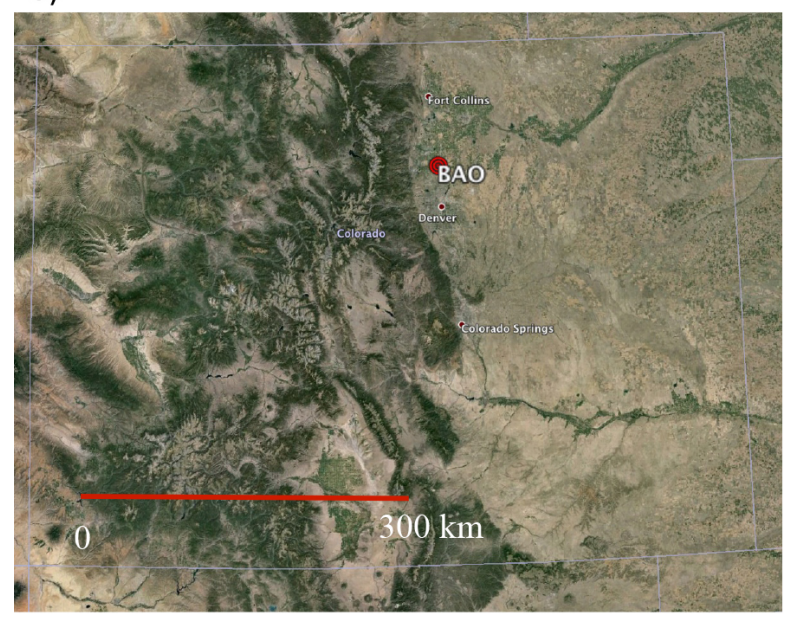

b)

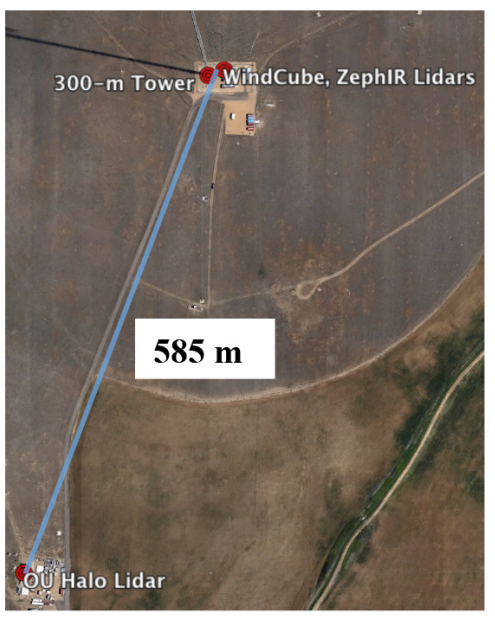

Figure 1. (a) Google Earth image of the state of Colorado. Location of BAO site is denoted by red marker. (b) Google Earth image of the BAO site. Instrument locations are denoted by red markers. Approximate distance between instruments is indicated by blue line and label. Only the initial location of the WindCube lidar is shown.

\subsection{Lower Atmospheric Thermodynamics and Turbulence Experiment (LATTE)}

LATTE was conducted from 10 February to 28 March 2014, with a small-scale extension of the project from 28 March to 28 April 2014. LATTE was conducted at the BAO, a National Oceanic and Atmospheric Administration (NOAA) facility located in Erie, Colorado (Fig. 1a). The BAO site is situated approximately $25 \mathrm{~km}$ east of the foothills of the Rocky Mountains. Although the diurnal heating cycle can induce upslope and downslope winds in the vicinity of a mountain range (e.g., Defant, 1951), these effects are only expected to influence flow at the BAO when the synoptic-scale pressure gradient is weak (Hahn, 1981). During LATTE, winds were primarily northerly and westerly throughout the lower boundary layer and appeared to be mainly associated with the upper-level flow pattern.

One of the primary goals of LATTE was to evaluate the accuracy of lidar turbulence measurements. Thus, the $300 \mathrm{~m}$ tower at the BAO was instrumented with three-dimensional sonic anemometers at six different heights. As a result of a collaboration with the National Center for Atmospheric Research (NCAR), NorthWest Research Associates, and the NOAA Earth System Research Laboratory (ESRL), we were able to mount two sonic anemometers at each measurement height on opposite booms such that at each height there would be at least one set of three-dimensional sonic anemometer measurements that were not strongly influenced by the wake of the tower. A Halo lidar owned by the University of Oklahoma (OU) along with a WindCube v2 and ZephIR 300 lidar, both owned by Lawrence Livermore National Laboratory (LLNL), were deployed at the BAO for LATTE, in addition to several instruments owned by NCAR. The OU Halo lidar was located approximately $600 \mathrm{~m}$ south- southwest of the $300 \mathrm{~m}$ tower so that it could be used to verify wind speeds from an NCAR wind profiler. The WindCube was located in the same enclosure as the $300 \mathrm{~m}$ tower from 14 to 28 February 2014, then moved to the same location as the OU Halo lidar from 1 March to 28 April 2014. The ZephIR remained in the tower enclosure for the duration of the experiment (Fig. 1b).

\subsection{Lower Atmospheric Boundary Layer Experiment (LABLE) 2}

LABLE took place in two phases: LABLE 1 was conducted from 18 September to 13 November 2012 and LABLE 2 was conducted from 12 June to 2 July 2013. LABLE 2 was a multi-lidar experiment designed to test different scanning strategies and will be discussed in this work. Detailed information on the research goals and instrumentation of LABLE can be found in Klein et al. (2015). Both LABLE campaigns took place at the central facility of the Southern Great Plains ARM site. The ARM site is operated by the Department of Energy and serves as a field site for an extensive suite of various in situ and remote sensing instruments (Mather and Voyles, 2013). The location of the ARM site in northern Oklahoma is shown in Fig. 2a.

Locations of the lidars deployed during LABLE 2 are shown in Fig. 2b. The ARM Halo lidar is a scanning lidar operated by the ARM site and is nearly identical to the OU Halo lidar. The Galion lidar is a lidar rented by OU that has identical hardware to the two scanning Halo lidars. Data from three-dimensional sonic anemometers on a $60 \mathrm{~m}$ tower were also available at the ARM site but could not be used to verify the six-beam lidar measurements as the tower was too short to overlap with the scanning lidar measurement heights (first range gate is $105 \mathrm{~m}$ ). Data from the $60 \mathrm{~m}$ sonic anemometer 
a)

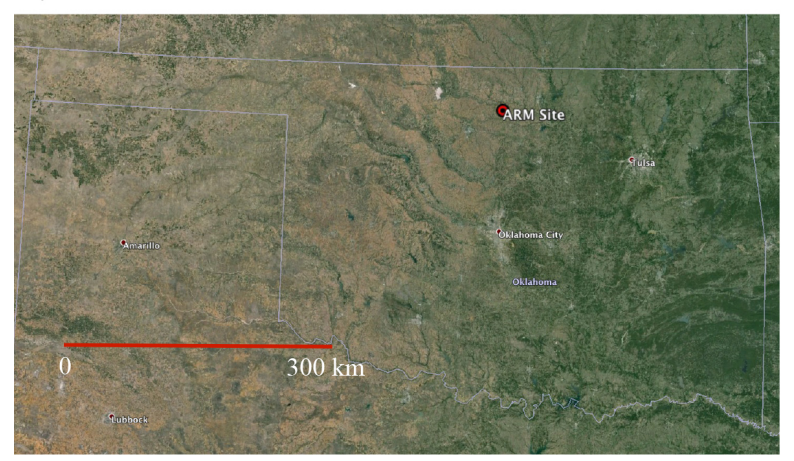

b)

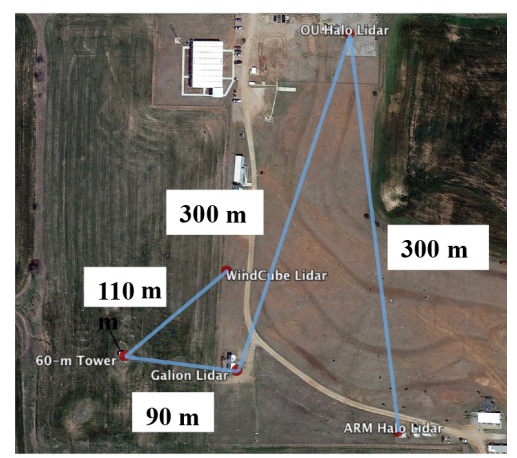

Figure 2. (a) Google Earth image of the state of Oklahoma. Location of Southern Great Plains ARM site is denoted by red marker. (b) Google Earth image of the central facility of the Southern Great Plains ARM site. Instrument locations are denoted by red markers. Approximate distances between instruments are indicated by blue lines and labels.

could be directly compared to corresponding measurements from the WindCube lidar, which has a first range gate of $40 \mathrm{~m}$, so only data from the WindCube lidar are shown in this work. Results from the scanning lidar portion of LABLE 2 are presented in Newman et al. (2016).

\section{Data processing}

\subsection{Coordinate rotation}

A coordinate rotation was applied to the sonic anemometer and lidar data to reduce the effects of alignment and tilt errors on the variance estimates (Foken, 2008). Following the procedure outlined by Kaimal and Finnigan (1994), the coordinate axes were first rotated such that the mean meridional wind speed, $\bar{v}$, was set to 0 and $u$ was aligned with the $10 \mathrm{~min}$ mean wind direction. In the next step, the coordinate axes were rotated such that $\bar{w}$ was equal to 0 .

Typically, the coordinate rotation is applied to the raw wind speed components before the variance is calculated, such that the variance is also defined in the new coordinate system. However, instead of first rotating the raw wind speed components, the variance values themselves from the old coordinate system can also be rotated such that $u$ is aligned with the mean wind direction and $\bar{v}$ is forced to 0 , as in Sathe et al. (2015). The rotated variance components are described as follows:

$\overline{u^{\prime 2}}$ rot $=\overline{u^{\prime 2}} \sin ^{2} \Theta+\overline{v^{\prime 2}} \cos ^{2} \Theta+\overline{u^{\prime} v^{\prime}} \sin 2 \Theta$,

${\overline{v^{\prime 2}}}_{\text {rot }}=\overline{u^{\prime 2}} \cos ^{2} \Theta+\overline{v^{\prime 2}} \sin ^{2} \Theta-\overline{u^{\prime} v^{\prime}} \sin 2 \Theta$,

${\overline{w^{\prime 2}}}_{\text {rot }}=\overline{w^{\prime 2}}$,

where $\Theta$ is the mean wind direction and the subscript rot refers to variance components in the rotated coordinate system. This rotation has the same effect as applying the first coordinate rotation to the original wind speed components before taking the variance. Thus, in comparisons with the six-beam technique, only the first coordinate rotation was applied to the lidar and sonic data to be consistent with the coordinate rotation used by Sathe et al. (2015).

It was determined during our analysis that the covariance term $\overline{u^{\prime} v^{\prime}}$ in Eqs. (32)-(33) is also affected by variance contamination when a DBS or VAD scan is used, in addition to the $u$ and $v$ variance components. At the BAO, variance contamination caused WindCube values of $\overline{u^{\prime} v^{\prime}}$ to differ largely from the sonic values under unstable conditions. However, the $\overline{u^{\prime} v^{\prime}}$ term measured by the sonics at both sites was nearzero throughout the day and only had a negligible effect on the coordinate rotations for the sonic variance. Thus, the $\overline{u^{\prime} v^{\prime}}$ term was neglected in Eqs. (32) and (33) for rotation of the lidar data.

\subsection{Quality control}

The actual sampling frequencies of the sonic anemometers and lidars drifted slightly around their prescribed sampling frequencies throughout the measurement campaigns, which is problematic for the calculation of variance. Thus, the raw wind speed data from the different instruments were linearly interpolated onto temporal grids with constant spacing that matched the sampling frequency of each instrument $(1 \mathrm{~Hz} / 0.25 \mathrm{~Hz}$ for the WindCube $\mathrm{v} 2,0.067 \mathrm{~Hz}$ for the ZephIR, $0.033 \mathrm{~Hz}$ for the Halo lidar, and $30 \mathrm{~Hz}(60 \mathrm{~Hz})$ for the north (south) sonic anemometers at the BAO tower). The sonic anemometer data were additionally averaged to form $10 \mathrm{~Hz}$ data streams. The $10 \mathrm{~Hz}$ data streams were used in further calculations, as they served to reduce high-frequency noise in the sonic anemometer data as well as reduce processing time. (Values of the $30 \mathrm{~min}$ variance calculated from the $10 \mathrm{~Hz}$ data streams did not differ significantly from values calculated from the raw sonic anemometer data streams.) The $60 \mathrm{~m}$ sonic anemometer data at the ARM site were also interpolated to a $10 \mathrm{~Hz}$ grid. No averaging was needed for the ARM sonic data, as the output frequency of the ARM sonic anemometers is already $10 \mathrm{~Hz}$ (Table 1 ). 
The spike filter developed by Højstrup (1993) and adapted by Vickers and Mahrt (1997) was used to flag outliers in the data. A 10 min window was shifted through the raw lidar and sonic anemometer data, and any point that was more than 3.5 standard deviations from the 10 min block average was flagged as a possible spike and removed from the data set. This process was repeated until no more spikes were detected. For each pass through the spike filter, the factor of 3.5 standard deviations was increased by 0.1 standard deviations.

By default, WindCube radial velocities that were associated with signal-to-noise ratios (SNRs) lower than $-23 \mathrm{~dB}$ were flagged as missing values. For the scanning lidars, SNR thresholds were set to -23 and $-17 \mathrm{~dB}$ for the horizontal and vertical beams, respectively. The ZephIR lidar obtains an estimate of the mean noise level by taking measurements with the shutter closed before each full scan. Only signals with power that exceeds a threshold of 5 standard deviations above this mean noise level are used to estimate the velocity (Slinger and Harris, 2012).

As Doppler lidars use the Doppler shift from aerosols to estimate radial velocity, they are adversely affected by the presence of precipitation particles, which can result in beam attenuation and increased vertical velocities (e.g., Huffaker and Hardesty, 1996; Pearson et al., 2009). Thus, lidar data that were collected when rain gauges at the different field sites recorded precipitation were flagged as erroneous data.

\subsection{Selection of averaging times}

In order to mitigate the effects of random errors on variance calculations, Mahrt et al. (1996) and Vickers and Mahrt (1997) recommend averaging products of perturbations over a period of time that is longer than the local averaging length, $T$, the averaging time that is used to calculate mean values from which the perturbations are derived. In this work, the variance of each velocity component was defined as the mean value of $u_{i}^{\prime 2}$ (calculated using $T=10 \mathrm{~min}$ ) over a $30 \mathrm{~min}$ period, with $i=1,2,3$ corresponding to the $u, v$, and $w$ estimates, respectively. The typical averaging period for wind energy studies is $10 \mathrm{~min}$, but a $30 \mathrm{~min}$ averaging period was used in this work to reduce the effects of noise on variance estimates, as in Sathe et al. (2015). The variance calculated with this method is hereafter referred to as the " 30 min variance", although it differs from the standard calculation of $30 \mathrm{~min}$ variance. These variance estimates represent turbulent motions with timescales from $0.01 \mathrm{~s}$ to $10 \mathrm{~min}$, with the smallest scales of turbulence only measured by the sonic anemometers.

Mesoscale motions can also induce errors in variance calculations, as the mean of each variable can change significantly over the averaging period used to calculate variance as a result of a frontal passage or wind direction shift (Vickers and Mahrt, 1997). Thus, raw wind speed data were detrended using a linear detrend method for each hour-long record. The detrending method served to reduce high variance values that were associated with large shifts in wind speed or wind direction.

\subsection{Stability classification}

At the BAO, temperature and wind speed data were available at multiple heights on the tower, so the gradient Richardson number, $\mathrm{Ri}$, was used as a stability parameter. Ri is defined by the following equation (Arya, 2001):

$R i=\frac{g}{T_{o}} \frac{\frac{\partial \theta}{\partial z}}{\left(\frac{\partial U}{\partial z}\right)^{2}}$,

where $g\left(\mathrm{~m} \mathrm{~s}^{-2}\right)$ is the gravitational acceleration, $T_{o}(\mathrm{~K})$ is the surface temperature, and $\frac{\partial U}{\partial z}\left(\mathrm{~s}^{-1}\right)$ and $\frac{\partial \theta}{\partial z}\left(\mathrm{Km}^{-1}\right)$ are the vertical gradients of horizontal wind speed and potential temperature, respectively. In this work, the potential temperature gradient was approximated by adding the dry adiabatic lapse rate, $\Gamma_{\mathrm{d}}$, to the temperature gradient, and the derivatives of temperature and wind speed were approximated by using a finite differencing approach, similar to the procedure used by Bodine et al. (2009):

$R i=\frac{g\left[\left(T_{z 2}-T_{z 1}\right) / \Delta z_{T}+\Gamma_{\mathrm{d}}\right] \Delta z_{U}^{2}}{T_{z 1}\left(U_{z 2}-U_{z 1}\right)^{2}}$,

where $z 1$ and $z 2$ correspond to two different measurement heights, and $\Delta z_{T}$ and $\Delta z_{U}$ refer to the differences in measurement levels for $T$ and $U$. As wind shear was often extremely low during the daytime hours at the BAO, a bulk wind shear quantity was used in Eq. (36), i.e., $z_{1}=0 \mathrm{~m}$ was assumed for wind speed, with $U_{z 1}=0 \mathrm{~ms}^{-1}$. This bulk approximation eliminated the extremely large negative $\mathrm{Ri}$ values that were often produced at the BAO under unstable conditions as a result of the small difference between $U_{z 2}$ and $U_{z 1}$.

Due to unexpected tower maintenance at the ARM site, it was not often possible to measure the temperature and wind speed at two heights simultaneously. Thus, the MoninObukhov length, $L(\mathrm{~m})$, from the $60 \mathrm{~m}$ sonic was used to define stability instead. $L$ is defined by the following equation:

$L=-\frac{u_{*}^{3} \overline{\theta_{v}}}{\kappa g \overline{w^{\prime} \theta_{v}^{\prime}}}$

where $u_{*}\left(\mathrm{~m} \mathrm{~s}^{-1}\right)$ is the friction velocity, $\overline{\theta_{v}}(\mathrm{~K})$ is the mean virtual potential temperature at the measurement height, $\kappa$ is the von Kármán constant (commonly set to 0.4 ), and $\overline{w^{\prime} \theta_{v}^{\prime}}$ $\left(\mathrm{m} \mathrm{s}^{-1} \mathrm{~K}\right)$ is the heat flux measured at the surface (e.g., Arya, 2001). Negative values of both $L$ and $\mathrm{Ri}$ indicate unstable conditions while positive values indicate stable conditions. As the data sets analyzed in this work are relatively small, only a broad classification of conditions as either stable or unstable was made. 


\section{Comparison of turbulence parameters: LATTE}

Figure 3 demonstrates the typical diurnal cycle of turbulence $\left(\sigma_{u}^{2}, \sigma_{v}^{2}\right.$, and $\left.\sigma_{w}^{2}\right)$ at the BAO, with low values of turbulence occurring during the evening and overnight hours (approximately 00:00-12:00 UTC) and high values of turbulence occurring during daytime, convective conditions (approximately 12:00-00:00 UTC). (Note that for all LATTE plots, data from the NCAR sonics are shown, unless the mean wind direction corresponded to the NCAR sonic wake sector, in which case the OU sonics were used. Local time is UTC-7.) During this period, the wind direction generally shifted between easterly/southeasterly and northerly (Fig. 3e). However, from approximately 18:00 UTC 23 March to 06:00 UTC 24 March, winds were primarily from the west/northwest, which is the direction of the Rocky Mountains. Flow from the mountains was associated with higher mean wind speeds and variances of the $u$ and $v$ velocity components in comparison to the rest of the period (Fig. 3).

The following sections focus on measurements from 25 March 2014, which was a calm, clear day with no precipitation when all three lidars had good data availability. Variance estimates from each lidar and scanning strategy are compared to similar measurements made by the sonic anemometers and the other lidars. For most comparison plots, variance estimates from the measurement height where the lidar data availability was greatest are shown. For the WindCube and ZephIR lidars, which only collect measurements up to 200 ma.g.l., data from $100 \mathrm{~m}$ a.g.l. are shown. For the Halo lidar, which has a minimum range gate of $105 \mathrm{~m}$, data from 200 ma.g.l. are shown.

\subsection{DBS technique: WindCube}

During the overnight hours of 25 March, variance values computed from the WindCube DBS data agreed well with sonic anemometer data, but between 15:00 and 21:00 UTC the WindCube substantially overestimated the $u$ and $v$ variance (Fig. 4a and b). Sathe et al. (2011) attribute this overestimation to variance contamination, which artificially increases the lidar-measured variance and is most prominent under unstable conditions, when the effects of volume averaging are minimized due to the relatively large turbulent eddy sizes. In Sect. 2.4, we presented a framework that further details the causes of variance contamination errors and provides equations for correcting variances computed from lidar DBS scans. These equations are now evaluated using sonic and lidar data.

\subsubsection{Variance correction}

As discussed in Sect. 2.4, instantaneous velocity values calculated from lidar DBS data contain extra terms, for example, the $\mathrm{d} v$ and $\mathrm{d} w$ terms in Eq. (15), which become large under
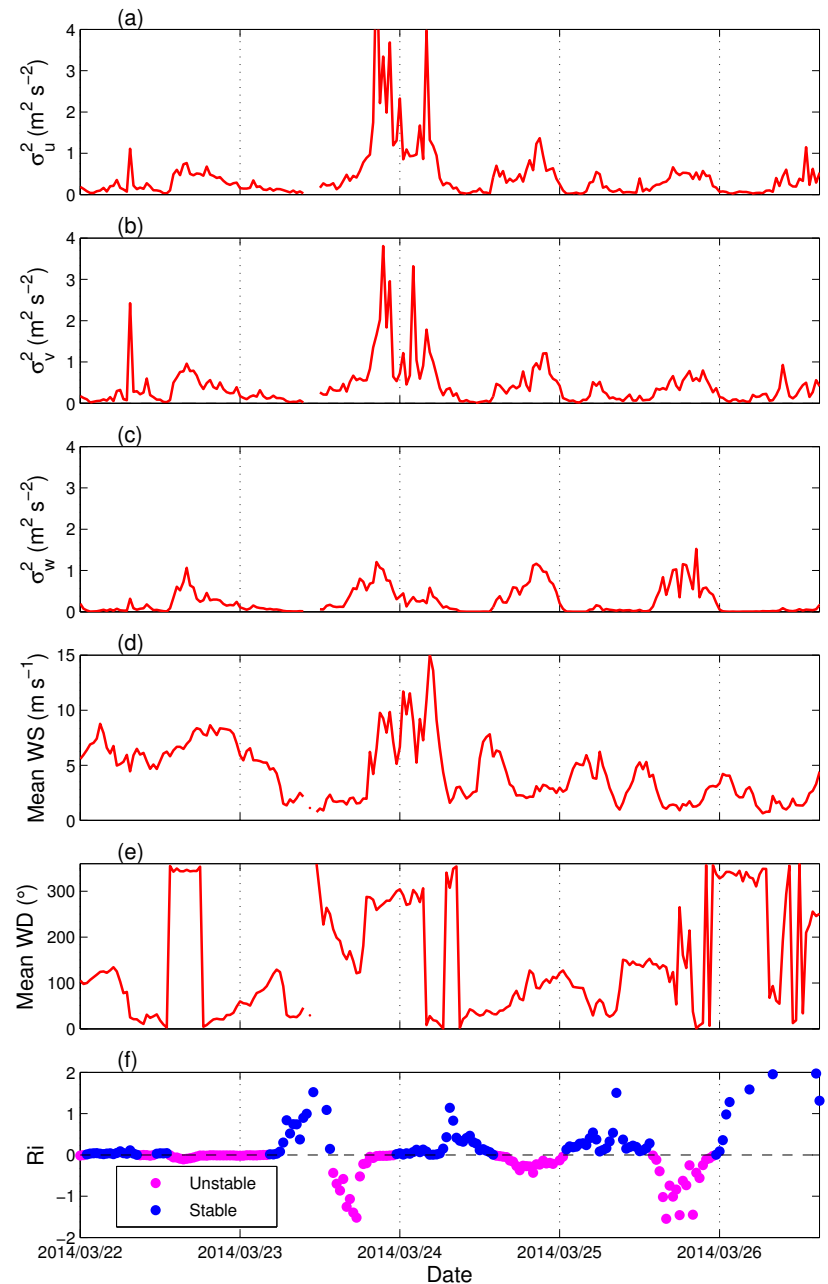

Figure 3. $30 \mathrm{~min}$ (a) $u$ variance, (b) $v$ variance, (c) $w$ variance, (d) mean wind speed, and (e) mean wind direction at $100 \mathrm{~m}$ from sonic anemometers at BAO and (f) Richardson number calculated from tower data. Data are shown from 22 to 26 March 2014, and tick marks for each date correspond to 00:00 UTC on that day.

convective conditions. The addition of these extra terms can cause the WindCube to overestimate the magnitude of the instantaneous $u$ and $v$ velocity and contaminate the true value of the variance, as shown in Eqs. (23) and (24) and seen in Fig. $4 a, b$. Since these extra terms cannot be easily quantified from lidar data, sonic anemometer data were used to examine the impacts of temporal and spatial changes in the instantaneous velocity components on the resultant variance estimates.

At the BAO tower, two sonic anemometers were located approximately $11.5 \mathrm{~m}$ apart on opposite booms at each measurement height, which were used to simulate the measurement technique used by the WindCube lidar. First, sonic data were projected into the directions of the WindCube beam positions and projected data from the south sonic were shifted forward in time by $2 \mathrm{~s}$ to simulate the time it takes the Wind- 

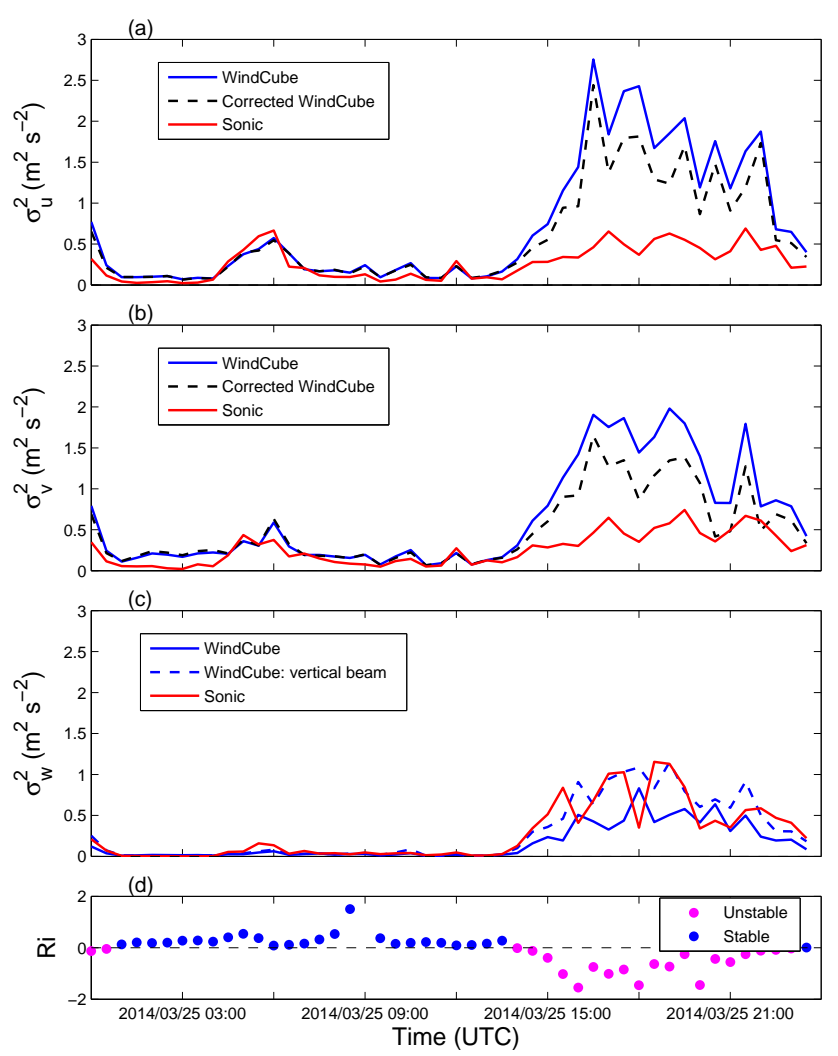

Figure 4. $30 \mathrm{~min}$ (a) $u$ variance, (b) $v$ variance, and (c) $w$ variance at $100 \mathrm{~m}$ from sonic anemometers and WindCube DBS technique at BAO and (d) Richardson number calculated from tower data. Data are shown from 25 March 2014. In (a) and (b), solid blue line indicates DBS-calculated variance and dashed black line indicates corrected variance. In (c), solid blue line indicates DBS-calculated $w$ variance and dashed blue line indicates $w$ variance calculated from vertically pointing beam. The corrected $u$ and $v$ variance data show substantial improvement over the uncorrected data set during unstable conditions.

Cube lidar beam to move from one side of the scanning circle to the other. The time-shifted and projected sonic data were then used to calculate values of $\rho_{u}, \rho_{v}$, and $\rho_{w}$.

Sonic anemometer data from 22 to 26 March 2014 were used to estimate values of the autocorrelation functions for times when neither sonic was waked by the tower. Mean values of $\rho_{u}, \rho_{v}$, and $\rho_{w}$ under unstable conditions were 0.96 , 0.81 , and 0.66 , respectively. Under stable conditions, the average values were $0.95,0.71$, and 0.69 . These values indicate that the $u, v$, and particularly $w$ wind components do change significantly in both space and time and that values of $w$ become decorrelated more quickly than values of $u$ and $v$ as a result of the presence of smaller turbulent scales of motion in the vertical direction.

The mean values of $\rho_{u}, \rho_{v}$, and $\rho_{w}$ calculated from the sonic data were then used with Eqs. (23) and (24) to correct the WindCube DBS variance values, where the value of $\overline{w^{\prime 2}}$ in the correction equations was taken to be the velocity vari- ance measured by the WindCube vertical beam. Corrected $u$ and $v$ variance values on 25 March 2014 are indicated by the black dashed lines in Fig. 4. The variance correction does not significantly change variance values under stable conditions, when the value of $\overline{w^{\prime 2}}$ is small, but it serves to reduce estimates of $\overline{u^{\prime 2}}$ and $\overline{v^{\prime 2}}$ by over $20 \%$ under unstable conditions, bringing them closer to the values measured by the sonics. In reality, the values of the autocorrelation functions needed for the correction should be smaller than the values that were calculated for the sonics, as the sonics were only located $11.5 \mathrm{~m}$ apart while the WindCube scanning cone has a diameter of $106 \mathrm{~m}$ at a measurement height of $100 \mathrm{~m}$. Thus, there is still some variance contamination present in the corrected $u$ and $v$ variance values shown in Fig. 4 , as the values of the autocorrelation functions used in the correction do not fully represent the degree of decorrelation that occurs between the WindCube beams.

Another method to calculate values of the autocorrelation functions is to use a least-squares approach to find the values of $\rho_{u}, \rho_{v}$, and $\rho_{w}$ that provide the best estimate for the sonic variance. This method yielded similar values of $\rho_{u}$ and $\rho_{v}$ to the values calculated from the sonic data and much lower values of $\rho_{w}$ in comparison to the sonic values (approximately 0.29 under unstable conditions and 0.13 under stable conditions). True estimates of the autocorrelation functions for the distances spanned by the WindCube beams would require either sonic anemometers at different towers or a numerical model that provides wind speed data with high spatial and temporal resolution.

\subsubsection{Methods for estimating $w$ variance}

Contamination errors also affect the variance of the $w$ component if it is computed applying the DBS method, although it generally does not lead to variance overestimates, as volume averaging for the $w$ component is more significant than it is for the $u$ and $v$ components (Sathe et al., 2011). However, the WindCube $\mathrm{v} 2$ lidar utilizes a vertical beam position once per scan to obtain a direct measurement of the vertical velocity directly above the lidar, which is only minimally affected by variance contamination. Both the vertical beam method and the DBS method (Eq. 10) were investigated in this work to determine the advantage of having a vertically pointed beam position to measure $w$ variance. At the BAO, the $w$ variance measured by the WindCube lidar's vertical beam was much higher and more accurate than the $w$ variance calculated from the DBS equations, particularly under convective conditions (Fig. 4c). This is not surprising, as the vertical beam variance is a measure of the variance directly above the lidar (barring the effects of volume averaging), while the DBS-estimated variance is an average across the scanning circle. Thus, in all further plots, $w$ variance from the WindCube lidar is calculated from the vertical beam. 

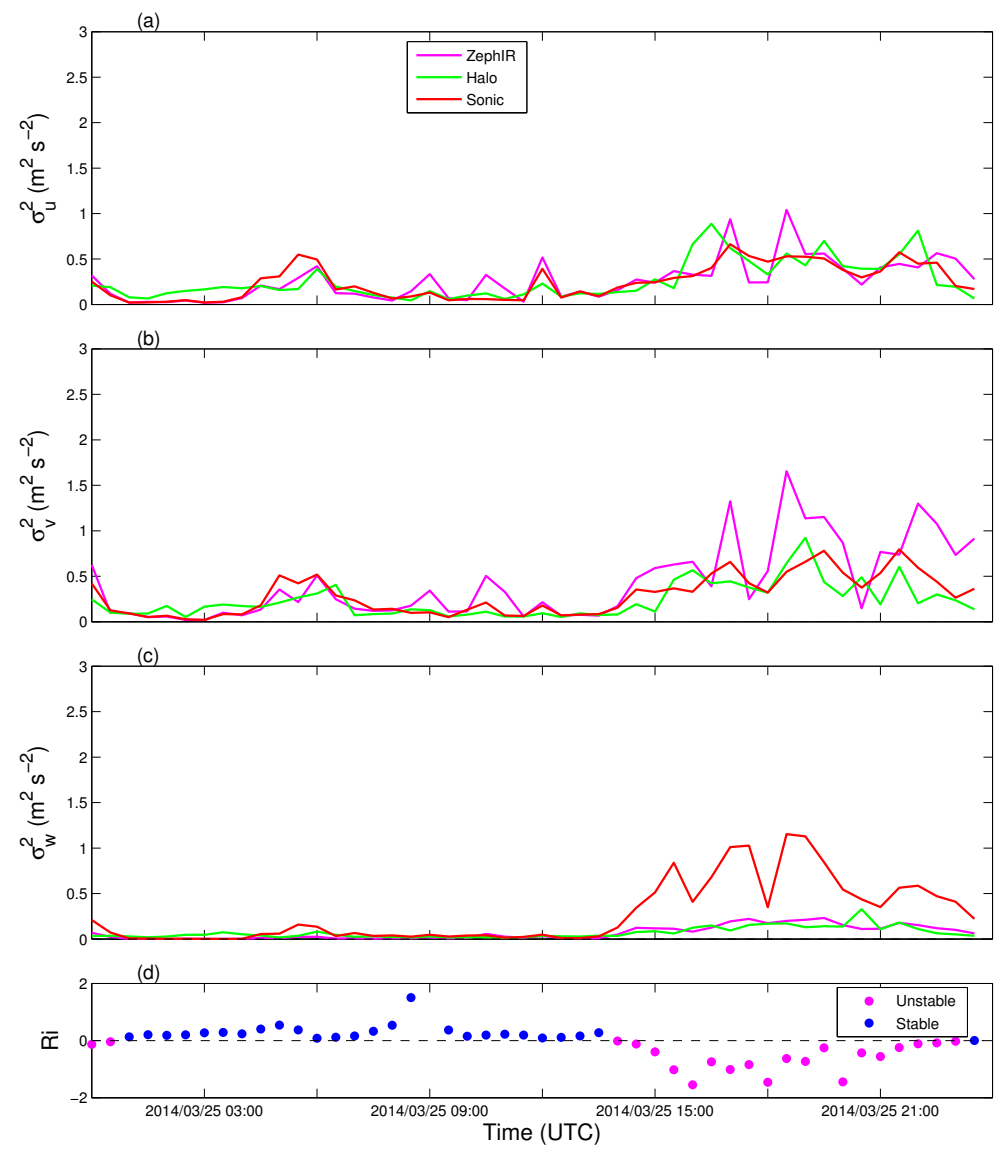

Figure 5. $30 \mathrm{~min}$ (a) $u$ variance, (b) $v$ variance, and (c) $w$ variance at $100 \mathrm{~m}$ from sonic anemometers and lidar VAD techniques at BAO and (d) Richardson number calculated from tower data. Data are shown from 25 March 2014. Comparison of the Halo and ZephIR VADprocessed data suggests that a lower elevation angle may be advantageous (e.g., lower $v$ variance values measured by the Halo lidar, which uses a lower elevation angle, shown in b). Note the smaller $u$ and $v$ variance values shown here for the Halo and ZephIR (VAD) as compared to the Wind Cube (DBS) in Fig. 4.

\subsection{VAD technique: ZephIR, Halo}

During post-processing, a VAD technique (Browning and Wexler, 1968) was used to calculate variance from the sixbeam Halo data. The five off-vertical beams were fit to a sine curve to estimate the horizontal wind speed, wind direction, and vertical wind speed from each scan. This information was then used to create a time series for the $u, v$, and $w$ components from which the variance could be calculated. Variance from the Halo VAD technique was compared to the variance estimated by the ZephIR lidar, which employs a 50point VAD at each height as part of its scanning strategy, as well as variance measured by the sonic anemometers.

While the ZephIR-estimated $u$ variance values were quite close to those measured by the sonic anemometers and the Halo lidar (Fig. 5a), the ZephIR overestimated the $v$ variance under unstable conditions during some half-hourly periods (Fig. 5b), which could indicate that the ZephIR lidar VAD technique is also affected by variance contamination, similar to the WindCube lidar. Although the WindCube and
ZephIR lidars use similar elevation angles (Table 1), the overestimation of $v$ variance by the ZephIR lidar was not nearly as large as it was for the WindCube lidar. The ZephIR has variable range gate sizes and takes nearly 4 times as long to complete a full scan from 10 to $200 \mathrm{~m}$ as the WindCube lidar, so the lower temporal resolution of the ZephIR scans may have caused it to measure lower variance values than the WindCube lidar. The Halo lidar produced the most accurate VAD-estimated $u$ and $v$ variance values throughout the day (Fig. 5a and b), suggesting that a VAD technique with a lower elevation angle can measure horizontal variance values more accurately. The Halo lidar used an elevation angle of $45^{\circ}$ while the WindCube and ZephIR lidars used elevation angles of $62^{\circ}$ and $60^{\circ}$, respectively. Although the values of $\mathrm{d} v$ and $\mathrm{d} w$ were likely larger for the Halo lidar, since it used a wider scanning cone, the contribution of the $\overline{\mathrm{d} w^{2}}$ term to the $v$ variance in Eq. (16) is smaller for lower values of $\phi$. Additionally, the temporal resolution of the Halo lidar likely led to the measurement of lower variance values than the Wind- 

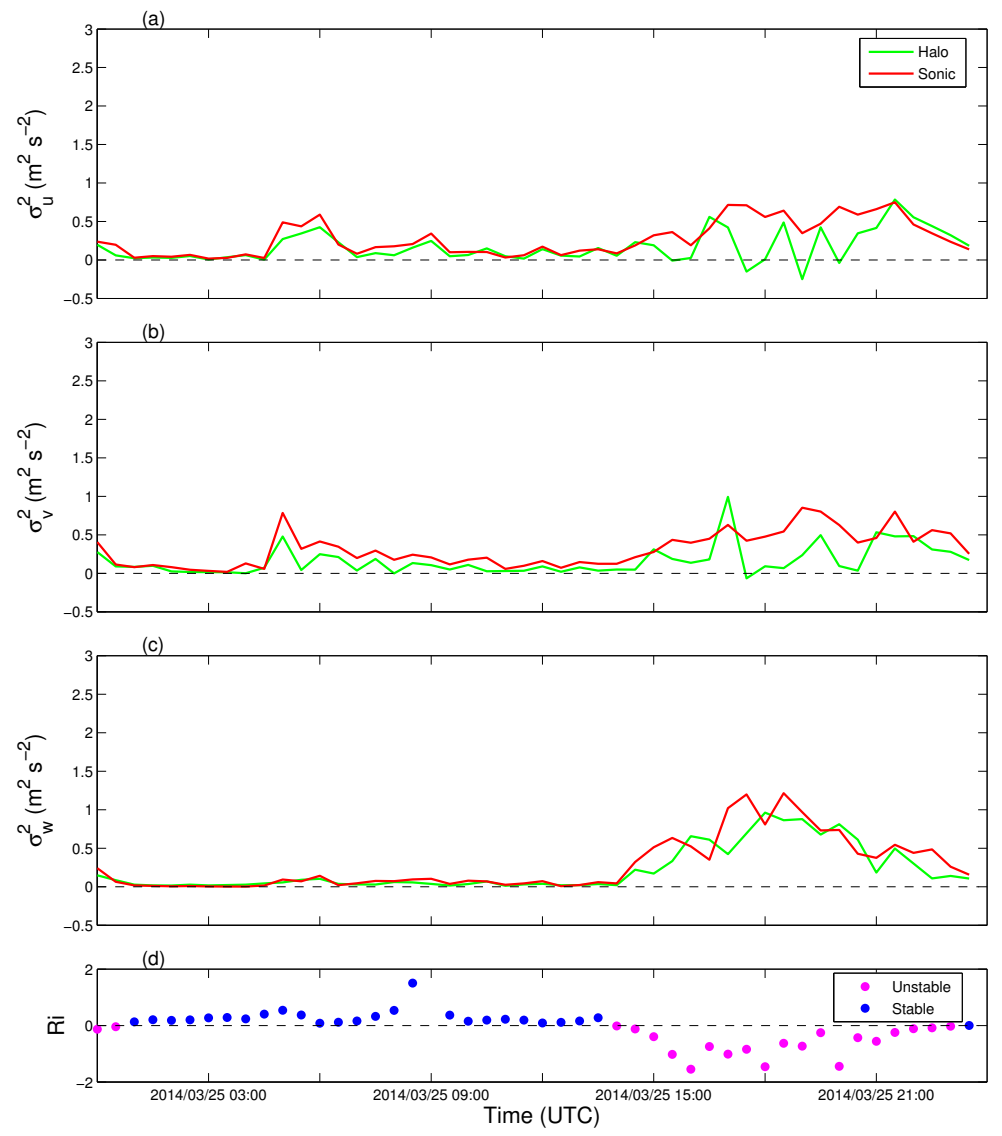

Figure 6. Thirty-minute (a) $u$ variance, (b) $v$ variance, and (c) $w$ variance at $200 \mathrm{~m}$ from sonic anemometers and Halo lidar six-beam technique at BAO and (d) Richardson number calculated from tower data. Data are shown from 25 March 2014. Note the high agreement between the six-beam Halo method and sonic in (c) as compared to the VAD Halo method in Fig. 5c for $w$ variance. (The difference in vertical variance accuracy is also likely related to the different turbulent scales present at 100 and $200 \mathrm{~m}$.)

Cube and ZephIR lidars, which may have masked the effects of variance contamination.

The ZephIR and Halo lidars measured similar $w$ variance values with the VAD technique, which were underestimates in comparison to the sonic anemometer values for nearly all stability conditions throughout the day (Fig. 5c). As previously discussed, the most accurate lidar method for measuring the $w$ variance appears to be the use of a vertical beam position to obtain a direct measurement of the vertical wind speed (Figs. $4 \mathrm{c}$ and $6 \mathrm{c}$ ).

\subsection{Halo: six-beam technique}

Variance measured using the six-beam technique with the Halo lidar is compared to variance measured by the sonic anemometers in Fig. 6. Similar to the WindCube lidar, the six-beam technique includes a vertically pointed beam to obtain a direct measurement of the vertical velocity. Vertical variance estimated by the Halo six-beam technique was much higher and more accurate than the vertical variance measured by the Halo VAD technique (Figs. 5c and 6c).
However, larger discrepancies occurred in the $u$ and $v$ variance values. During strongly unstable conditions from 17:00 to 21:00 UTC, the Halo six-beam technique often underestimated the $u$ and $v$ variance in comparison to the sonic anemometers (Fig. 6a and b). In some extreme cases, the $u$ and $v$ variance values became negative, which should be mathematically impossible given the definition of variance $\left(\sigma_{u_{i}}^{2}=\overline{\left(u_{i}-\overline{u_{i}}\right)^{2}}\right)$.

In order to determine the cause of this horizontal variance underestimation and the negative variance values, it is instructive to examine the equations used to calculate the variance components with the six-beam technique (Eqs. 29-31). Equations (29) and (30) for the $u$ and $v$ variance, respectively, both include the term $-\overline{v_{\mathrm{r} 6}^{\prime}}$, meaning that the variance calculated from the vertical beam radial velocity is subtracted from the combination of the other terms. Thus, when $\overline{v_{\mathrm{r} 6}^{\prime 2}}$ is large, as is often the case under convective conditions (Fig. 6c), or overestimated due to instrument noise, a large value is subtracted in Eqs. (29) and (30), and the $u$ and $v$ variance can become negative if the other radial variances are not measured 

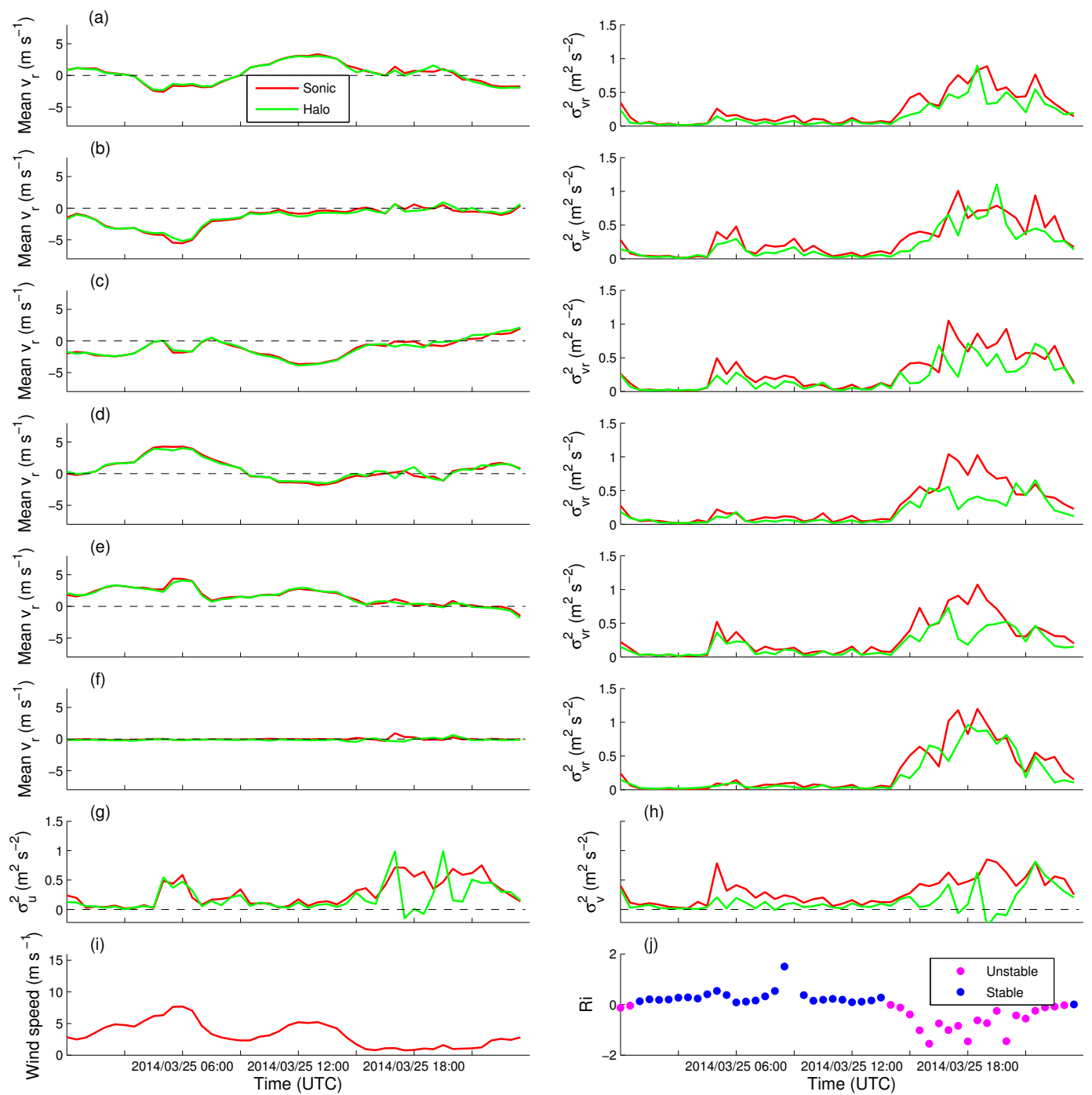

(h)

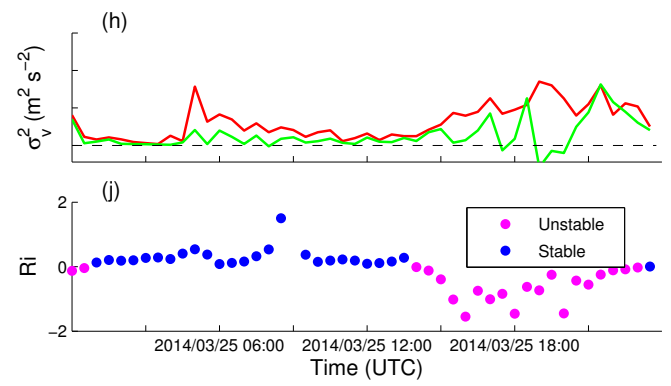

Figure 7. 30 min mean velocity values (left panels) and variance values (right panels) for (a) $v_{\mathrm{r} 1}$, (b) $v_{\mathrm{r} 2}$, (c) $v_{\mathrm{r} 3}$, (d) $v_{\mathrm{r} 4}$, (e) $v_{\mathrm{r} 5}$, and (f) $v_{\mathrm{r} 6}$ measured by Halo lidar and calculated from projected sonic data. Values of (g) $u$ variance and (h) $v$ variance are also shown for reference, where sonic values are from standard variance calculation and Halo values are from six-beam calculation. Mean wind speed from sonic is shown in (i) and Richardson number from tower is shown in (j). Data are shown from 25 March 2014 at 200 ma.g.l. at the BAO tower.

accurately. The other negative terms in Eqs. (29) and (30) could also decrease the horizontal variance components and cause them to become negative. Similarly, if the positive terms in Eqs. (29) and (30) are underestimated, the variance values would also likely be underestimated. Although negative values of $\sigma_{u}^{2}$ and $\sigma_{v}^{2}$ only comprised approximately $5 \%$ of the horizontal variance values at $200 \mathrm{~m}$ during the 5-day analysis period, the underestimation of horizontal variance components by the six-beam technique is a significant issue that warrants further investigation.

Velocity data from the $200 \mathrm{~m}$ sonic anemometers were projected into the directions of the different Halo beam locations in order to assess the accuracy of the measurements from each beam position. Time series plots of the 30 min mean radial wind speeds and radial variance values measured by the sonics and Halo lidar on 25 March 2014 are shown in Fig. 7. During the afternoon of 25 March, mean wind speeds were very low (Fig. 7i), which is reflected by the low radial wind speeds measured by the Halo lidar and calculated from the projected sonic data (Fig. 7a-f). Some minor differences in the radial wind speeds measured by the Halo and sonic anemometer were evident in the late afternoon, as well as strongly underestimated and negative Halo $u$ and $v$ variance values (Fig. $7 \mathrm{~g}$ and $\mathrm{h}$ ). The largest discrepancies between the radial variance values also occurred in the late afternoon, when the Halo strongly underestimated the variance of the radial velocity at the third, fourth, and fifth beam positions (Fig. 7c-e). In the initial six-beam equations, terms $\overline{v_{\mathrm{r} 3}^{\prime}}, \overline{v_{\mathrm{r} 4}^{\prime 2}}$, and $\overline{v_{\mathrm{r} 5}^{\prime 2}}$ have positive coefficients in the $u$ vari- 
ance equation (Eq. 29), and terms $\overline{v_{\mathrm{r} 3}^{\prime 2}}$ and $\overline{v_{\mathrm{r} 4}^{\prime 2}}$ have positive coefficients in the $v$ variance equation while the $\overline{v_{\mathrm{r} 5}^{\prime}{ }^{2}}$ term has a negative coefficient (Eq. 30). The actual coefficients of the radial beam variances will change once the coordinate rotation is applied (Eqs. 32-34), but for the most part weighted values of $\overline{v_{\mathrm{r} 3}^{\prime 2}}, \overline{v_{\mathrm{r} 4}^{\prime 2}}$, and $\overline{v_{\mathrm{r} 5}^{\prime 2}}$ are added to the weighted values of the other radial beam variances to obtain values for the $u$ and $v$ variance. Thus, if the variance measured at beam positions 3,4 , and 5 is underestimated, the $u$ and $v$ variance will also be underestimated. Similar trends were also observed on 24 March 2014 (not shown).

Several factors may have caused the Halo lidar to underestimate the variance at certain beam positions more strongly than at other beam positions. One possible explanation for the variance discrepancies could be the presence of horizontal heterogeneity across the lidar scanning circle. The six-beam technique requires the assumption that flow is homogeneous in the scanning circle encompassed by the five off-vertical beams, and this assumption may not have been valid at the BAO, which is located in the vicinity of complex terrain, especially at a measurement height of 200 ma.g.l. Horizontal heterogeneity and high values of variance could cause large amounts of scatter about the VAD sine curve (Weitkamp, 2005). The differences between the instantaneous radial velocities and the fit VAD sine curve (Eq. 25) were examined for 25 March, but no noticeable differences were evident for the different beam positions, although residuals were much larger under unstable conditions. A modeled flow field and lidar simulator would likely be needed to definitively quantify the effect of horizontal heterogeneity on the variances measured by the different lidar beam positions.

Relative intensity noise (RIN) also may have affected the variance values measured by the Halo lidar on 25 March. RIN results from spontaneous radiation emissions from the laser, which cause intensity fluctuations in the laser oscillator (Chang, 2005). In a coherent heterodyne lidar, RIN appears as pink noise; i.e., it is mainly present in the low-frequency part of the Doppler spectrum (Courtney et al., 2008). Since low wind speeds would also be detected in the low frequency part of the spectrum, RIN can impact the accuracy of Doppler velocity measurements under low wind speeds. Peña et al. (2009) found that a ZephIR lidar most strongly underestimated the turbulence intensity measured by cup anemometers when weak wind speeds were measured.

As several of the Halo radial beams measured radial wind speeds that were close to $0 \mathrm{~m} \mathrm{~s}^{-1}$ during the afternoon of 25 March (Fig. 7a-f), it is possible that RIN caused the Halo lidar to underestimate the variance at certain beam positions. To further investigate this possibility, mean radial velocity and variance values were calculated for 6 March 2014, a date from the campaign when atmospheric conditions were less strongly convective and wind speeds were higher during the late afternoon (Fig. 8). Although there were some small biases in the radial wind speed measurements from the Halo li- dar (Fig. 8a-f), there were no large discrepancies in the radial variance measurements on 6 March and no strongly underestimated or negative $u$ and $v$ variance values (Fig. $8 \mathrm{~g}-\mathrm{h}$ ). This suggests that the six-beam technique is more accurate when wind speeds are higher, as radial variance estimates are more accurate under higher wind speed conditions and more accurate horizontal variance estimates are produced as a result. However, it is difficult to make this assessment with the limited data set available. For the 5-day period selected for this study, there was no clear trend between the mean radial wind speed measured at each beam location and the error in Halomeasured variance at each beam location. It should be noted that the mean wind speeds measured during the afternoon of 25 March rarely exceeded $3 \mathrm{~ms}^{-1}$, which is below the typical cut-in speed for a modern wind turbine (e.g., Burton et al., 2001). Thus, variance measurements under low wind speeds would likely not be used for wind energy applications.

\subsection{Application of six-beam technique to WindCube lidar}

A technique similar to the six-beam strategy can be applied to the WindCube data by substituting the DBS values of $\theta$ and $\phi$ into Eq. (3). The $\overline{u^{\prime} v^{\prime}}$ term drops out because either $\cos \theta$ or $\sin \theta$ is equal to 0 for every beam position, resulting in five equations and five unknowns. Similar to the Halo six-beam technique, these equations can be solved simultaneously to obtain values of the $u, v$, and $w$ variance, which can then be rotated into the coordinate system aligned with the mean wind.

Variance measured by the individual WindCube beams is compared to variance calculated from projected sonic data in Fig. 9. Similar to the Halo lidar (Figs. 7 and 8), the variance of the radial velocities was sometimes overestimated by the WindCube lidar and sometimes underestimated. Although there were no large discrepancies between the WindCube and sonic radial variance under unstable conditions (14:0023:00 UTC), the five-beam technique produced large $u$ and $v$ variance underestimates and several negative variance values (Fig. 9f and g). Thus, even when a lidar with better temporal resolution and a smaller scanning circle than the Halo lidar is used, the simultaneous use of all the radial beam velocity variances to calculate the $u$ and $v$ variance can result in large uncertainties, especially during unstable conditions.

In summary, at this site, the WindCube and Halo lidars were not able to measure the radial beam variances accurately enough to estimate the horizontal variance values with a five- or six-beam technique, possibly because wind speeds at the site were often too low to accurately measure variance with lidars. In the next section, the five-beam technique is evaluated at the ARM site, where mean wind speeds were much higher in comparison to the BAO. 

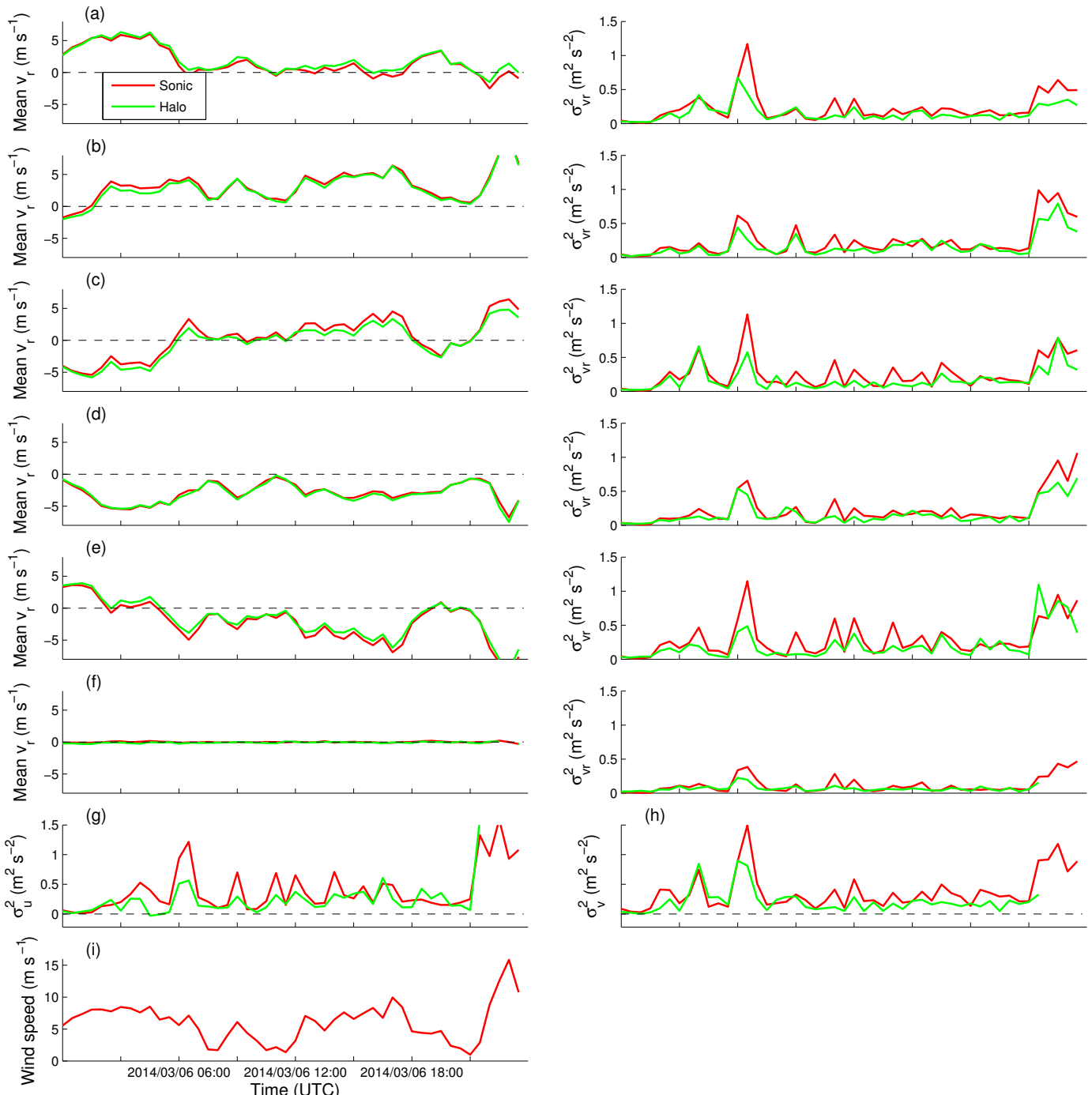

Figure 8. As in Fig. 7 but for 6 March 2014. Richardson number is not shown, because the $10 \mathrm{~m}$ temperature sensor had data quality issues on this day (possibly related to tower icing).

\subsection{Note on the effects of temporal resolution}

The temporal resolution between the sonic anemometers and the lidars at the BAO is drastically different; while the NCAR and OU sonics collect data at frequencies of 60 and $30 \mathrm{~Hz}$, respectively, the lidars collect data at a frequency of $1 \mathrm{~Hz}$, with most scanning strategies taking much longer than $1 \mathrm{~s}$. In order to examine the effect of temporal resolution on variance estimates, the sonic data streams were artificially degraded in temporal resolution and then used to calculate the threedimensional variance components. Temporal resolutions of $1,4,15$, and $30 \mathrm{~s}$ were selected to represent the time it takes the WindCube to update the wind vector, the time for a full WindCube scan, the time for a full ZephIR scan, and the time for a full Halo six-beam scan, respectively. On 25 March 2014 , the use of either 1 or $4 \mathrm{~s}$ temporal resolution resulted in percent errors around $5 \%$ for $u$ and $v$ and $10 \%$ for $w$ while the use of either 15 or $30 \mathrm{~s}$ resolution resulted in larger errors of 20 to $50 \%$ in the variance estimates (not shown). Thus, the temporal resolution of the lidar scans likely influenced the variance estimates in addition to the scanning strategy used, particularly for the ZephIR and Halo lidars.

\section{Comparison of turbulence parameters: LABLE 2}

Plots of the 30 min variance, mean wind speed and direction, and Monin-Obukhov length from the $60 \mathrm{~m}$ sonic over 5 days at the ARM site are shown in Fig. 10. Diurnal trends in the LABLE 2 turbulence parameters are similar to those seen in the LATTE data: turbulence is fairly low during overnight, stable conditions before increasing during daytime, convective conditions. However, mean wind speeds at the ARM site 

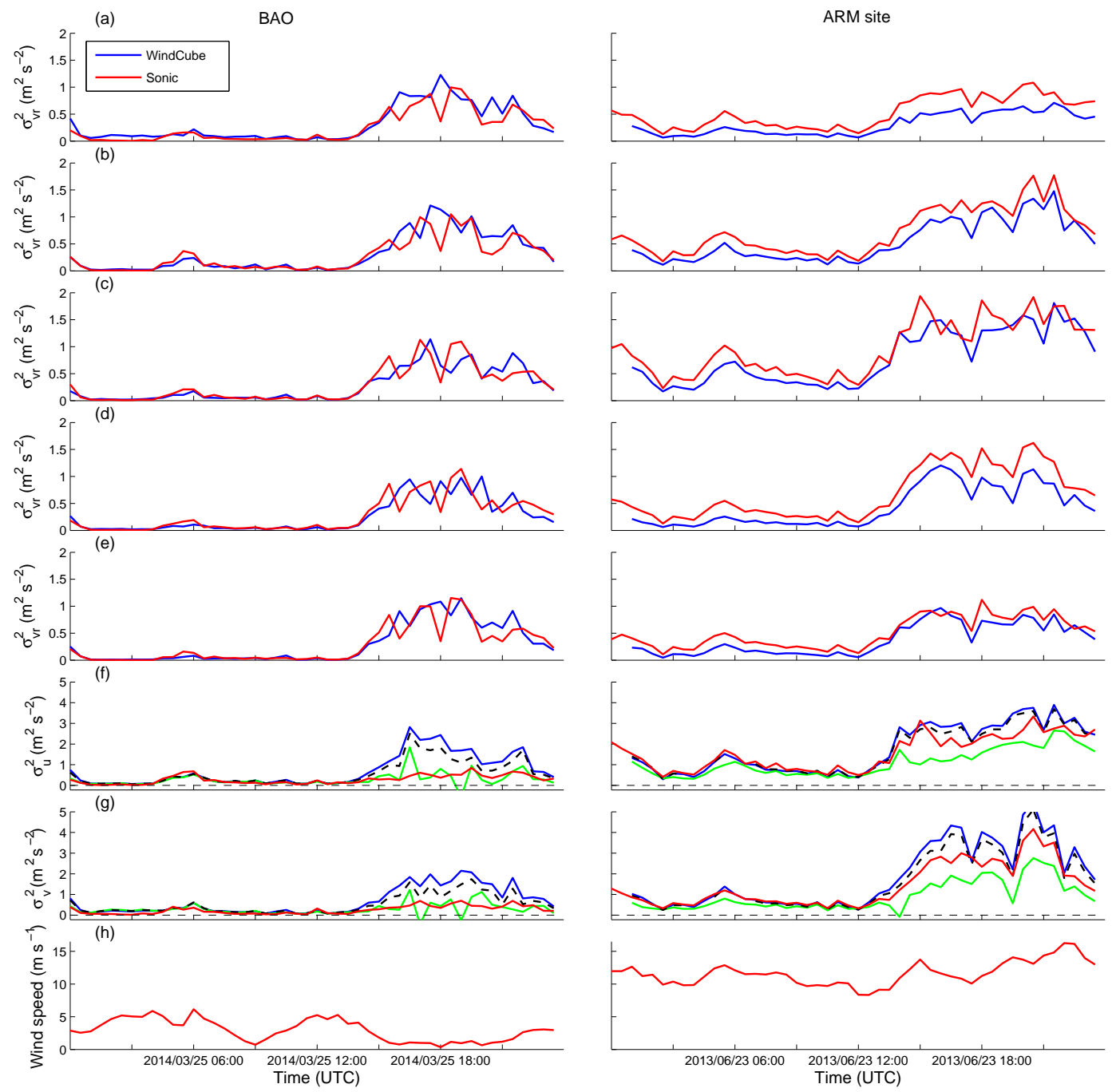

Figure 9. Thirty-minute (a) $v_{\mathrm{r} 1}$, (b) $v_{\mathrm{r} 2}$, (c) $v_{\mathrm{r} 3}$, (d) $v_{\mathrm{r} 4}$, and (e) $v_{\mathrm{r} 5}$ variance values measured by WindCube lidar and calculated from projected sonic data, where positions $1-4$ are located at azimuths of $0,90,180$, and $270^{\circ}$, respectively, at an elevation angle of $62^{\circ}$ and position 5 is pointed vertically. Values of (f) $u$ variance and (g) $v$ variance are also shown for reference, where sonic values are from standard variance calculation and WindCube values are shown from DBS calculation (solid blue line), five-beam calculation (green line), and corrected DBS calculation (dashed black line). Mean wind speed from sonic anemometer is shown in (h). Data are shown from the BAO on 25 March 2014 at 100 ma.g.l. (left panels) and from the ARM site on 23 June 2013 at 60 ma.g.l. (right panels). The WindCube five-beam method results in large underestimates of $u$ and $v$ variance, especially under convective conditions at the BAO, while much better agreement is seen with the new variance correction method.

were generally much higher than at the BAO, and winds were nearly constantly from the south/southeast. In addition, variance values were generally much higher at the ARM site. In comparison to the BAO, the ARM site is located in much simpler terrain and SNR values tended to be much higher, so lidar variance measurements are expected to be more accurate.

Time series of 30 min radial variance values estimated at $60 \mathrm{~m}$ from projected sonic data and WindCube lidar data during LABLE 2 on 23 June 2013 are shown in the right panels of Fig. 9. In contrast to the case shown from the BAO (left panels in Fig. 9), variance values are higher throughout the day and the WindCube lidar nearly always underestimated the radial variance values calculated from the projected sonic data (Fig. 9a-e). However, when applying the DBS method, the WindCube again overestimated the $u$ and $v$ variance under unstable conditions as a result of variance contamination (Fig. 9f and g).

The five-beam method and the variance correction method described in Sect. 5.1.1 were also applied to the $60 \mathrm{~m}$ WindCube data at the ARM site. Values of $\rho_{u}, \rho_{v}$, and $\rho_{w}$ obtained from the sonics at the BAO were used in the variance correction equations (Eqs. 23-24) to determine how well this correction worked at a different site. At the ARM site, both 

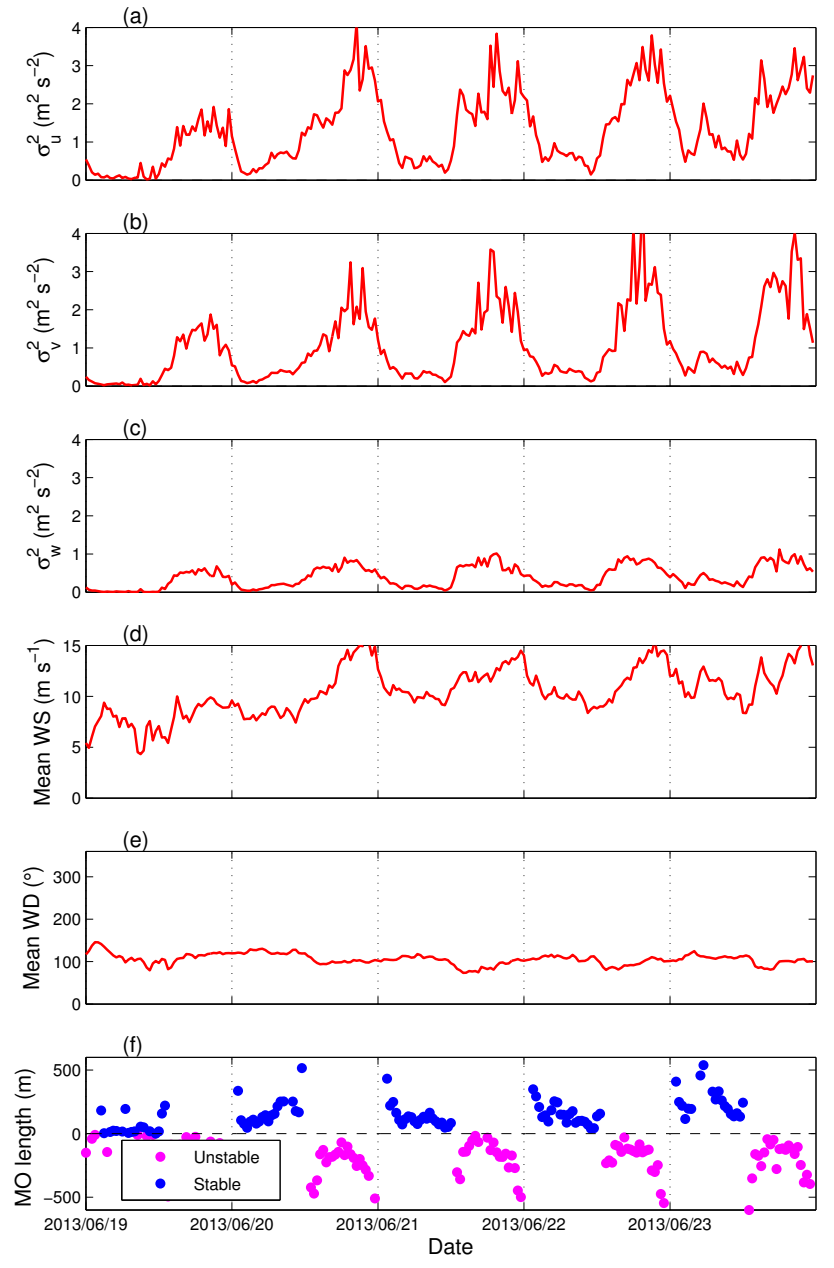

Figure 10. Thirty-minute (a) $u$ variance, (b) $v$ variance, (c) $w$ variance, (d) mean wind speed, (e) mean wind direction, and (f) Monin-Obukhov length at $60 \mathrm{~m}$ from sonic anemometer at the ARM site. Data are shown from 19 to 23 June 2013, and tick marks for each date correspond to 00:00 UTC on that day.

the five-beam method and variance correction method produced nearly identical variance values under stable conditions, while the five-beam method produced much lower variance values under unstable conditions (Fig. 9f, g). However, none of the five-beam $u$ and $v$ variance values were negative, in contrast to the BAO data. This likely occurred because the WindCube underestimated the variance from the radial velocities by approximately the same amount throughout the day. In particular, the WindCube measured lower $w$ variance values than the sonic anemometer during nearly all time periods on 23 June 2013 at the ARM site (Fig. 9e, right panel) while the WindCube measured $w$ variance values that were approximately the same as or slightly higher than those measured by the sonic anemometers at the BAO (Fig. 9e, left panel). Similar to the six-beam equations, the value of the vertical variance is subtracted from the sum of the other terms in the five-beam equations. As discussed in Sect. 5.3, when the value of the vertical variance is large or the vertical variance is overestimated, this can cause the calculated $u$ and $v$ variance to become negative. This did not occur at the ARM site, as values of the vertical variance were much smaller than variance values from the other beam positions under unstable conditions, in contrast to the BAO, where the vertical variance values were similar to the variance estimated from the horizontal radial beams. Thus, the vertical variance term had a much larger influence on the $u$ and $v$ variance values for the example shown at the BAO than it did at the ARM site.

Velocity spectra from the two sites were calculated in order to examine the scales of turbulence measured at the different locations. Averaged spectra for unstable conditions are shown in Fig. 11 for the $60 \mathrm{~m}$ ARM sonic data and the 50 and $100 \mathrm{~m}$ BAO sonic data. The spectral power calculated from the ARM site data is much higher than for the BAO data, which is reflected by the higher values of variance measured at the ARM site in comparison to the BAO (Figs. 3 and 10). The largest difference between the spectral shapes occurs for the $w$ spectra, where the peak in the ARM site spectrum is shifted to higher frequencies in comparison to the BAO spectra at both 50 and $100 \mathrm{~m}$. Thus, the vertical turbulent scales present during the campaign at the ARM site appear to be generally smaller than those measured at the BAO site under unstable conditions. This caused the WindCube lidar to often underestimate the $w$ variance at the ARM site, as the effects of temporal resolution and volume averaging are more significant for smaller turbulent scales. SNR values were also generally higher at the ARM site than at the BAO, so lower amounts of noise in the raw velocity data also likely led to the measurement of smaller vertical variance values. In addition, differences in season and measurement site characteristics may have affected the turbulent scales observed at the two sites. Higher mean wind speeds at the ARM site (Fig. 9h) also likely led to more accurate variance values in comparison to the BAO.

\section{Summary and conclusions}

The VAD and DBS scanning strategies, a novel correction method for the DBS strategy, and the six-beam lidar scanning strategy (Sathe, 2012) were evaluated at two measurement sites: the Southern Great Plains ARM site and the Boulder Atmospheric Observatory. As a $300 \mathrm{~m}$ tower with 12 sonic anemometers was located at the BAO, the evaluation primarily focused on data collected there.

One of the primary disadvantages of using a VAD or DBS technique with a high scanning elevation angle is the variance contamination that can occur as a result of differences in the instantaneous velocity at different parts of the scanning circle. In our work, the VAD and DBS techniques often measured variance values that were 60-80\% larger than those measured by a sonic anemometer as a result of variance 

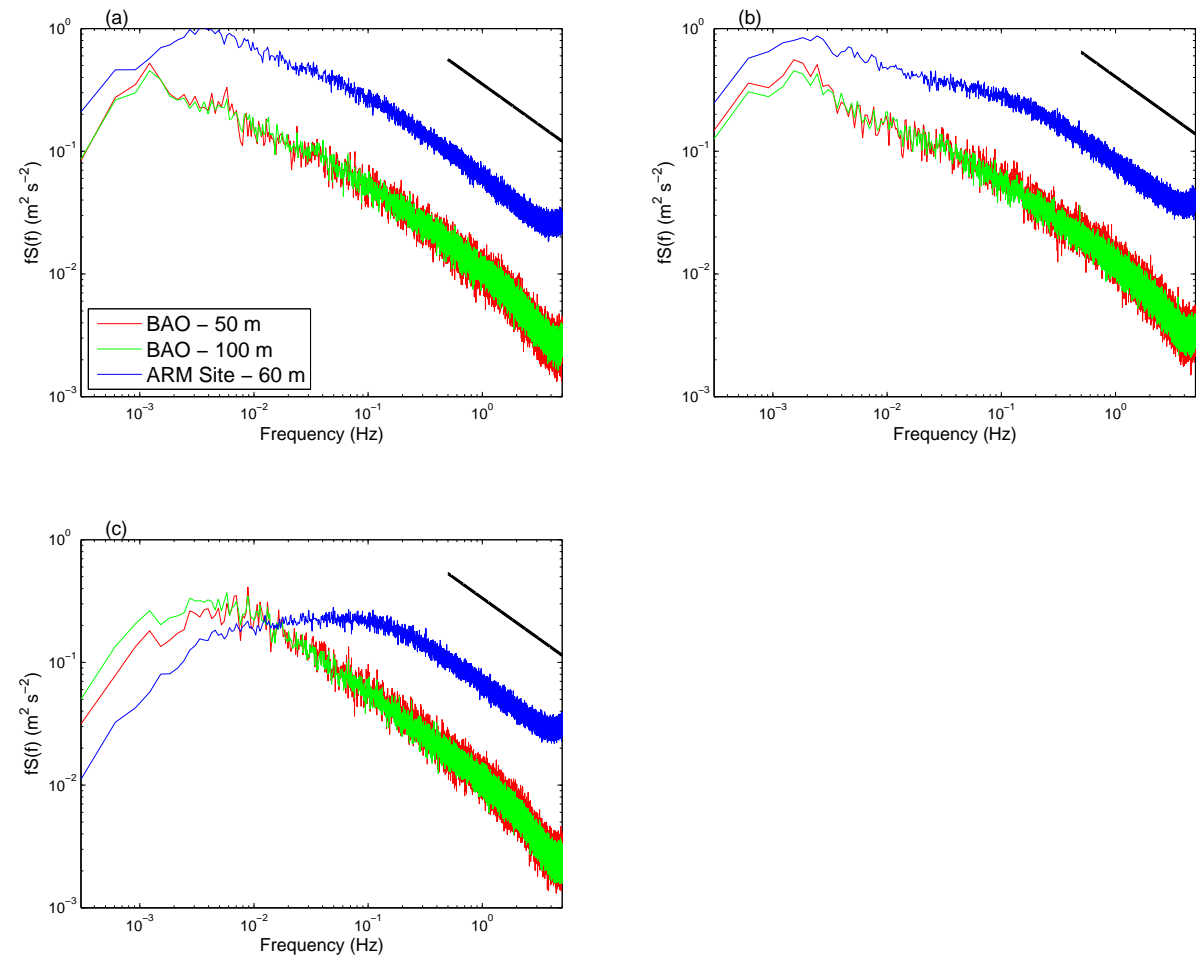

Figure 11. Averaged (a) $u$ spectra, (b) $v$ spectra, and (c) $w$ spectra for unstable conditions measured by the south BAO sonic at 50 and $100 \mathrm{~m}$ and the ARM sonic at $60 \mathrm{~m}$. Black line denotes theoretical $-2 / 3$ slope for inertial subrange. Note the larger amount of energy contained at the lowest frequency scales at the BAO for the $w$ component at both measurement heights.

contamination. Although using a smaller scanning cone mitigates the effects of horizontal heterogeneity on wind speed estimates, it also increases the contribution of variance contamination (Eqs. 23-24). In this work, a method was developed to correct DBS-estimated variance values for contamination. The additional variance terms were quantified using estimates of the autocorrelation functions $\rho_{u}, \rho_{v}$, and $\rho_{w}$ from sonic anemometer data at the BAO and vertical variance measured by the WindCube's vertical beam. This correction method was not expected to completely eliminate variance contamination, as estimations of the autocorrelation functions from the sonic anemometers were not fully representative of the decorrelation that actually occurs across the DBS scanning circle. In addition, it was assumed that turbulent fluxes remain constant across the scanning circle, which is not always true. However, the correction method still reduced WindCube variance overestimates by over $20 \%$ under unstable conditions at both the BAO and the ARM site. The correction method can be applied to other lidars that have a vertical beam position and does not require the use of a scanning lidar or complex calculations. Thus, it is a method that can be easily used by wind farm managers or researchers with commercially available lidars.

Another way to reduce variance contamination is to combine the radial velocity variance values and solve a set of equations to calculate the variance. This method was sug- gested by Sathe (2012) as a scanning strategy with six beam positions, and it can also be applied to the five beam positions used by the WindCube lidar. At the BAO, the calculation of horizontal variance with the five- and six-beam equations often led to variance underestimates and even negative $u$ and $v$ variance values (Figs. 6 and 9). The technique appears to be strongly affected by inaccurate variance measurements from one or more beam positions, which could be due to the low wind speeds and low SNR values measured at the BAO. At the ARM site, wind speeds were much higher and the WindCube lidar nearly always underestimated the radial velocity variances, likely as a result of the smaller turbulent scales present at the ARM site. The uniform underestimation of the radial velocity variances around the scanning circle led to more accurate five-beam variance estimates at the ARM site.

Acknowledgements. The authors would like to thank the staff of the Southern Great Plains ARM site, Tim Lim from NCAR, Lucas Root from NorthWest Research Associates, Shiril Tichkule from the University of Colorado at Boulder, Bruce Bartram and Daniel Wolfe from NOAA/ESRL's Physical Sciences Division, Marc Fischer and Sebastien Biraud from Lawrence Berkeley National Laboratory, the Boundary Layer Integrated Sensing and Simulation group at OU, and the technical support staff at Campbell Scientific, Leosphere, and Halo Photonics for their assistance during the experiments. We would also like to acknowledge the efforts of two 
reviewers, whose comments and suggestions helped improve the manuscript. LABLE 2 data were obtained from the Atmospheric Radiation Measurement (ARM) Climate Research Facility, a US Department of Energy Office of Science user facility sponsored by the Office of Biological and Environmental Research. J. F. Newman and S. Wharton received funding from the Laboratory Directed Research and Development (LDRD) award number 12-ERD-069 from the Lawrence Livermore National Laboratory. Livermore National Laboratory is operated by Lawrence Livermore National Security, LLC, for the US Department of Energy, National Nuclear Security Administration, under contract DE-AC52-07NA27344.

Edited by: M. Rapp

\section{References}

Arya, S. P.: Introduction to Micrometeorology, vol. 79 of International Geophysics Series, 2nd edn., Academic Press, Cornwall, UK, 2001.

Barthelmie, R. J., Crippa, P., Wang, H., Smith, C. M., Krishnamurthy, R., Choukulkar, A., Calhoun, R., Valyou, D., Marzocca, P., Matthiesen, D., Brown, G., and Pryor, S. C.: 3-D wind and turbulence characteristics of the atmospheric boundary layer, B. Am. Meteorol. Soc., 95, 743-756, 2013.

Bingöl, F., Mann, J., and Foussekis, D.: Conically scanning lidar error in complex terrain, Meteorol. Z., 18, 189-195, 2009.

Bodine, D., Klein, P. M., Arms, S. C., and Shapiro, A.: Variability of surface air temperature over gently sloped terrain, J. Appl. Meteorol. Clim., 48, 1117-1141, 2009.

Bright, D. R. and Mullen, S. L.: The sensitivity of the numerical simulation of the Southwest monsoon boundary layer to the choice of PBL turbulence parameterization in MM5, Weather Forecast., 17, 99-114, 2002.

Browning, K. A. and Wexler, R.: The determination of kinematic properties of a wind field using Doppler radar, J. Appl. Meteorol., 7, 105-113, 1968.

Burton, T., Sharpe, D., Jenkins, N., and Bossanyi, E.: Wind Energy Handbook, Wiley, West Sussex, UK, 2001.

Chang, W. S.: Principles of Lasers and Optics, Cambridge University Press, New York, NY, USA, 2005.

Clark, T. L., Hall, W. D., Kerr, R. M., Middleton, D., Radke, L., Ralph, F. M., Neiman, P. J., and Levinson, D.: Origins of aircraftdamaging clear-air turbulence during the 9 December 1992 Colorado downslope windstorm: numerical simulations and comparison with observations, J. Atmos. Sci., 57, 1105-1131, 2000.

Clifton, A. and Wagner, R.: Accounting for the effect of turbulence on wind turbine power curves, J. Phys. Conf. Ser., 524, 012109 , doi:10.1088/1742-6596/524/1/012109, 2014.

Collier, C. G., Davies, F., Bozier, K. E., Holt, A. R., Middleton, D. R., Pearson, G. N., Siemen, S., Willetts, D. V., Upton, G. J. G., and Young, R. I.: Dual-Doppler lidar measurements for improving dispersion models, B. Am. Meteorol. Soc., 86, 825-838, 2005.

Courtney, M., Wagner, R., and Lindelöw, P.: Commercial lidar profilers for wind energy: A comparative guide, in: European Wind Energy Conference, 1 April 2008, Brussels, Belgium, 2008.

Defant, F.: Local Winds, in: Compendium of Meteorology, American Meteorological Society, Boston, MA, USA, 655-672, 1951.
Foken, T.: Micrometeorology, Springer, Heidelberg, Germany, 2008.

Hahn, C.: A study of the diurnal behavior of boundary-layer winds at the Boulder Atmospheric Observatory, Bound.-Lay. Meteorol., 21, 231-245, 1981.

Højstrup, J.: A statistical data screening procedure, Meas. Sci. Technol., 4, 153-157, 1993.

Huffaker, R. M. and Hardesty, R. M.: Remote sensing of atmospheric wind velocities using solid-state and $\mathrm{CO}_{2}$ coherent laser systems, P. IEEE, 84, 181-204, 1996.

Kaimal, J. and Finnigan, J.: Atmospheric Boundary Layer Flows: Their Structure and Measurement, Oxford University Press, New York, NY, USA, 1994.

Kelley, N. D., Jonkman, B. J., and Scott, G. N.: The Great Plains turbulence environment: Its origins, impact and simulation., Tech. Rep., NREL/CP-500-40176., National Renewable Energy Laboratory, Golden, CO, USA, 2006.

Klein, P., Bonin, T. A., Newman, J. F., Turner, D. D., Chilson, P. B., Wainwright, C. E., Blumberg, W. G., Mishra, S., Carney, M., Jacobsen, E. P., Wharton, S., and Newsom, R. K.: LABLE: A multi-institutional, student-led, atmospheric boundary-layer experiment, B. Am. Meteorol. Soc., 96, 1743-1764, 2015.

Koscielny, A. J., Doviak, R. J., and Zrnic, D. S.: An evaluation of the accuracy of some radar wind profiling techniques, J. Atmos. Ocean. Tech., 1, 309-320, 1984.

Krishnamurthy, R., Boquet, M., and Machta, M.: Turbulence intensity measurements from a variety of Doppler lidar instruments, in: Proc. European Wind Energy Association Annual Meeting, 12 March 2014, Barcelona, Spain, PO.080, 2014.

Lhermitte, R. M. and Atlas, D.: Precipitation motion by pulse Doppler radar, in: Proc. Ninth Weather Radar Conference, Amer. Meteor. Soc., Boston, USA, 218-223, 1961.

Lundquist, J. K., Churchfield, M. J., Lee, S., and Clifton, A.: Quantifying error of lidar and sodar Doppler beam swinging measurements of wind turbine wakes using computational fluid dynamics, Atmos. Meas. Tech., 8, 907-920, doi:10.5194/amt-8-9072015, 2015.

Machta, M. and Boquet, M.: State of the art of measuring wind turbulence with pulsed lidars, in: European Wind Energy Association Annual Meeting, 5 February 2013, Vienna, Austria, 324PO, 2013.

Mahrt, L., Vickers, D., Howell, J., Højstrup, J., Wilczak, J. M., Edson, J., and Hare, J.: Sea surface drag coefficients in the Ris $\varnothing$ Air Sea Experiment, J. Geophys. Res.-Oceans, 101, 14327-14335, 1996.

Mather, J. H. and Voyles, J. W.: The ARM Climate Research Facility: a review of structure and capabilities, B. Am. Meteorol. Soc., 94, 377-392, 2013.

Newman, J. F., Bonin, T. A., Klein, P. M., Wharton, S., and Newsom, R. K.: Testing and validation of multi-lidar scanning strategies for wind energy applications, Wind Energy, accepted, doi:10.1002/we.1978, 2016.

Pearson, G., Davies, F., and Collier, C.: An analysis of the performance of the UFAM pulsed Doppler lidar for observing the boundary layer, J. Atmos. Ocean. Tech., 26, 240-250, 2009.

Peña, A., Hasager, C. B., Gryning, S.-E., Courtney, M., Antoniou, I., and Mikkelsen, T.: Offshore wind profiling using light detection and ranging measurements, Wind Energy, 12, 105-124, 2009. 
Peña, A., Hasager, C. B., Badger, M., Barthelmie, R. J., Bingöl, F., Cariou, J.-P., Emeis, S., Frandsen, S. T., Harris, M., Karagali, I., Larsen, S. E., Mann, J., Mikkelsen, T. K., Pitter, M., Pryor, S., Sathe, A., Schlipf, D., Slinger, C., and Wagner, R.: Remote Sensing for Wind Energy, Tech. Rep., DTU Wind Energy-E-Report-0084, Denmark Technical University, available at: http://orbit.dtu.dk/files/111814239/DTU_Wind_Energy_ Report_E_0084.pdf, last access: 17 November 2015.

Pichugina, Y. L., Tucker, S. C., Banta, R. M., Brewer, W. A., Kelley, N. D., Jonkman, B. J., and Newsom, R. K.: Horizontalvelocity and variance measurements in the stable boundary layer using Doppler lidar: Sensitivity to averaging procedures, J. Atmos. Ocean. Tech., 25, 1307-1327, 2008.

Sathe, A.: Influence of wind conditions on wind turbine loads and measurement of turbulence using lidars, $\mathrm{PhD}$ thesis, Delft University Wind Energy Research Institute, Delft, the Netherlands, 2012.

Sathe, A. and Mann, J.: Measurement of turbulence spectra using scanning pulsed wind lidars, J. Geophys. Res.-Atmos., 117, D01201, doi:10.1029/2011JD016786, 2012.

Sathe, A. and Mann, J.: A review of turbulence measurements using ground-based wind lidars, Atmos. Meas. Tech., 6, 3147-3167, doi:10.5194/amt-6-3147-2013, 2013.

Sathe, A., Mann, J., Gottschall, J., and Courtney, M. S.: Can wind lidars measure turbulence?, J. Atmos. Ocean. Tech., 28, 853-868, 2011.

Sathe, A., Mann, J., Vasiljevic, N., and Lea, G.: A six-beam method to measure turbulence statistics using ground-based wind lidars, Atmos. Meas. Tech., 8, 729-740, doi:10.5194/amt-8-729-2015, 2015.
Sjöholm, M., Mikkelsen, T., Mann, J., Enevoldsen, K., and Courtney, M.: Time series analysis of continuous-wave coherent Doppler lidar wind measurements, IOP. C. Ser. Earth. Env., 1, 012051, doi:10.1088/1755-1307/1/1/012051, 2008.

Slinger, C. and Harris, M.: Introduction to continuous-wave Doppler lidar, in: Summer School in Remote Sensing for Wind Energy, Boulder, USA, 11 June, 2012.

Strauch, R. G., Merritt, D. A., Moran, K. P., Earnshaw, K. B., and De Kamp, D. V.: The Colorado wind-profiling network, J. Atmos. Ocean. Tech., 1, 37-49, 1984.

Stull, R. B.: Meteorology for Scientists and Engineers, 2nd edn., Brooks/Cole, Pacific Grove, CA, USA, 2000.

Vickers, D. and Mahrt, L.: Quality control and flux sampling problems for tower and aircraft data, J. Atmos. Ocean. Tech., 14, 512 526, 1997.

Wainwright, C. E., Stepanian, P. M., Chilson, P. B., Palmer, R. D., Fedorovich, E., and Gibbs, J. A.: A time series sodar simulator based on large-eddy simulation, J. Atmos. Ocean. Tech., 31, 876889, 2014.

Weitkamp, C.: Lidar: Range-Resolved Optical Remote Sensing of the Atmosphere, Springer Series in Optical Sciences, Vol. 102, Springer, New York, NY, USA, 2005.

Westerhellweg, A., Cañadillas, B., Beeken, A., and Neumann, T.: One year of LiDAR measurements at FINO1-Platform: Comparison and verification to met-mast data, in: Proc. of the 10th German Wind Energy Conference DEWEK, 17-18 November, Bremen, Germany, 2010.

Wharton, S. and Lundquist, J. K.: Atmospheric stability affects wind turbine power collection, Environ. Res. Lett., 7, 014005, doi:10.1088/1748-9326/7/1/014005, 2012. 